\title{
Changes in traditional ecological knowledge of forage plants in immigrant villages of Ningxia, China
}

\author{
Ying Ma', Binsheng Luo ${ }^{1}$, Qiang Zhu², Dongxing Ma ${ }^{3}$, Qi Wen, Jinchao Feng ${ }^{1}$ and Dayuan Xue ${ }^{1 *}$
}

\begin{abstract}
Background: Ecological migration serves as an important measure for poverty eradication as well as for the protection, inheritance, and utilization of traditional ecological knowledge. This study investigated and cataloged the traditional forage plant resources and recorded the associated traditional knowledge of immigrant villages in Hongsibu District of Ningxia, China. The diversity of traditional forage plant resources and the changes in associated traditional ecological knowledge were compared among ecological immigrant villages from different emigration areas, with a hope of providing a reference for forage development, the conservation of wild forage plant resources, and the development of regional animal husbandry.
\end{abstract}

Methods: From March 2018 to May 2019, a field investigation was conducted in six villages in Ningxia. Through the snowball technique, a total of 315 immigrants were interviewed using various methods, including semistructured interviews and key person interviews, which included opportunities for free listing. The changes in the utilization of traditional forage plants were compared between the ecological migrants and the original inhabitants, and the causes underlying the changes were analyzed. In addition, the major forage plant species in the research area were investigated and evaluated.

Results: (1) The six investigated villages reported 224 traditional forage plant species that belong to 42 families and 150 genera. Compared with their original living areas, the number of traditional forage plant species used in the immigrant villages decreased with the increase in the relocation distance. (2) The utilization of traditional forage plants varied among the immigrants who moved to Hongsibu District from forest areas, loess hilly areas, and semiarid desertified areas. The smaller the difference was in ecological environment between the immigration and emigration areas, the more the traditional forage plant knowledge had been retained. (3) The diversity and associated knowledge of traditional forage plants retained by ecological migrants are closely correlated to gender, age, education level, and occupation.

Conclusion: This study revealed that the diversity of traditional forage plants and associated knowledge retained after migration vary among ecological immigrants from different areas; generally, the immigrants that relocated from a closer place retained more ecological knowledge. In the immigrant villages with significantly different natural resources and a long distance from the migrants' original locations, the diversity of traditional forage plants decreased, and the traditional knowledge about forage plants showed signs of being forgotten and abandoned by the younger generation. Therefore, measures are urgently needed to document and protect the forage plant resources and preserve the traditional knowledge of ecological immigrants.

Keywords: Ecological immigrant, Forage plant, Traditional knowledge, Hongsibu District, Ningxia

\footnotetext{
* Correspondence: xuedayuan@hotmail.com

${ }^{1}$ College of Life and Environmental Sciences, Minzu University of China,

Beijing 100081, People's Republic of China

Full list of author information is available at the end of the article
}

(c) The Author(s). 2019 Open Access This article is distributed under the terms of the Creative Commons Attribution 4.0 International License (http://creativecommons.org/licenses/by/4.0/), which permits unrestricted use, distribution, and reproduction in any medium, provided you give appropriate credit to the original author(s) and the source, provide a link to the Creative Commons license, and indicate if changes were made. The Creative Commons Public Domain Dedication waiver (http://creativecommons.org/publicdomain/zero/1.0/) applies to the data made available in this article, unless otherwise stated. 


\section{Background}

Based on the characteristics of local plant resources, local farmers and herdsmen inherit traditional knowledge of forage plants [1] and have a deep understanding of and practical experience with local forage resources [2]. This plays an important role in maintaining the positive development of agricultural and animal husbandry socioecological systems [3]. Local farmers' and herdsmen' traditional knowledge of forage plants, gained from local livelihoods and cultures that rely on natural herbage [4], plays an important role in regional biodiversity protection [5]. Farmers and herdsmen in areas with a long history of rearing livestock have a rich knowledge of forage plants, which has been recorded in the countries such as Morocco, West Africa, Brazil, Ethiopia, Pakistan, and Austria [2, 4, 6-10] to provide techniques and strategies for utilization, classification management, and sustainable development of forage plant resources. As a contracting party of the Convention on Biological Diversity, China has made active efforts to protect and record traditional knowledge associated with biodiversity and biological resources over the past two decades [11, 12]. Traditional knowledge for sustainable use of forage plants is indispensable and invaluable. For example, under China's policy of returning farmland to forests and grassland, farmers and herdsmen of Dulong (Drung or T'rung, an ethnic group in China) in the area of Yunnan Province, where available grassland resources are very limited, successfully maintained a sustainable agricultural system for livestock, forage plants, and crops by using their knowledge of native forage plants [7]. Animal husbandry is an ecological adaptation of humans living in grasslands. The herdsmen in Inner Mongolia of China have accumulated relevant knowledge regarding palatability and seasonal periodicity of forage plants, as well as livestock fatness status, which provides a good reference for the modernization of animal husbandry, such as intensive animal husbandry and the development of family farms [13].

Ecological migration is a phenomenon of population migration due to the interaction of ecological environment and other factor s[14-16]. In China, due to the needs of ecological civilization construction [17] and the aim of eliminating poverty [18], populations formerly living in those areas for designed nature preservation, or with severely damaged ecological environments, or ecologically fragile, or not suitable for human habitation, are requested to emigrate to other places for settlement. This is called ecological migration [14-18]. Ecological migration plays an important role in effectively alleviating environmental degradation [19], improving the living standards of immigrants, and developing local economy [20]. As an important measure of protecting biodiversity and reducing poverty [21], ecological migration results in a win-win situation to a certain extent. Ecological migration is of great value for preserving traditional cultures and knowledge related to biodiversity [22-25]. However, the separation of immigrants from their original natural resources and cultural atmosphere brings serious challenges regarding the preservation of traditional knowledge accumulated over generations [25], especially the inheritance of farmers and herdsmen's traditional knowledge of forage plants.

Ningxia is representative of an agro-pastoral transition zone [26] in China with massive ecological migration [27], where a thorough understanding regarding the connection and change between migrants and forage plant knowledge is particularly important. The forage plants in different ecological regions such as loess hilly regions, forest regions, and semiarid desertified regions in Ningxia have different characteristics. Therefore, traditional forage plant knowledge accumulated by farmers and herdsmen is regional, dependent, and adaptable. However, little attention has been paid to the impact of ecological migration on forage plant knowledge, and related research findings are scarce. Hence, further research on this topic is urgently needed. Through investigating the retention of traditional knowledge regarding forage plants and related livestock feeding experiences among ecological immigrants from different emigration areas in the Hongsibu District of Ningxia, this study aims to quantitatively analyze the changes in forage plant resources and the underlying influencing factors. Our findings may provide reference for the protection of forage plant resources and associated traditional knowledge during the implementation of ecological migration policy.

\section{Method}

\section{Research area}

The research was conducted in six villages in the Ningxia Hui Autonomous Region: four immigration villages in Hongsibu District, one emigration village in Jingyuan County, Guyuan Prefecture, and one emigration village in Haiyuan County, Zhongwei Prefecture (Fig. 1, Tables 1 and 2).

Hongsibu District of Wuzhong, Ningxia, is located in the hinterland of the arid zone in central Ningxia (between $105^{\circ} 43^{\prime}$ and $106^{\circ} 42^{\prime} \mathrm{E}, 37^{\circ} 28^{\prime}$ and $37^{\circ} 37^{\prime} \mathrm{N}$ ). The region is predominantly sandlands and grasslands in the grassland desertification control area in central Ningxia (Fig. 1). It has a dry climate with annual precipitation of 200-400 $\mathrm{mm}$. It is mainly covered by psammophytic steppe and desert steppe. In 1998, supported by the national Seven-Year Priority Poverty Reduction Program, the Yellow River Irrigation Project for Poverty Reduction in Ningxia ("1236" Project) was launched. As a result, Hongsibu District was formed by pumping water from the 


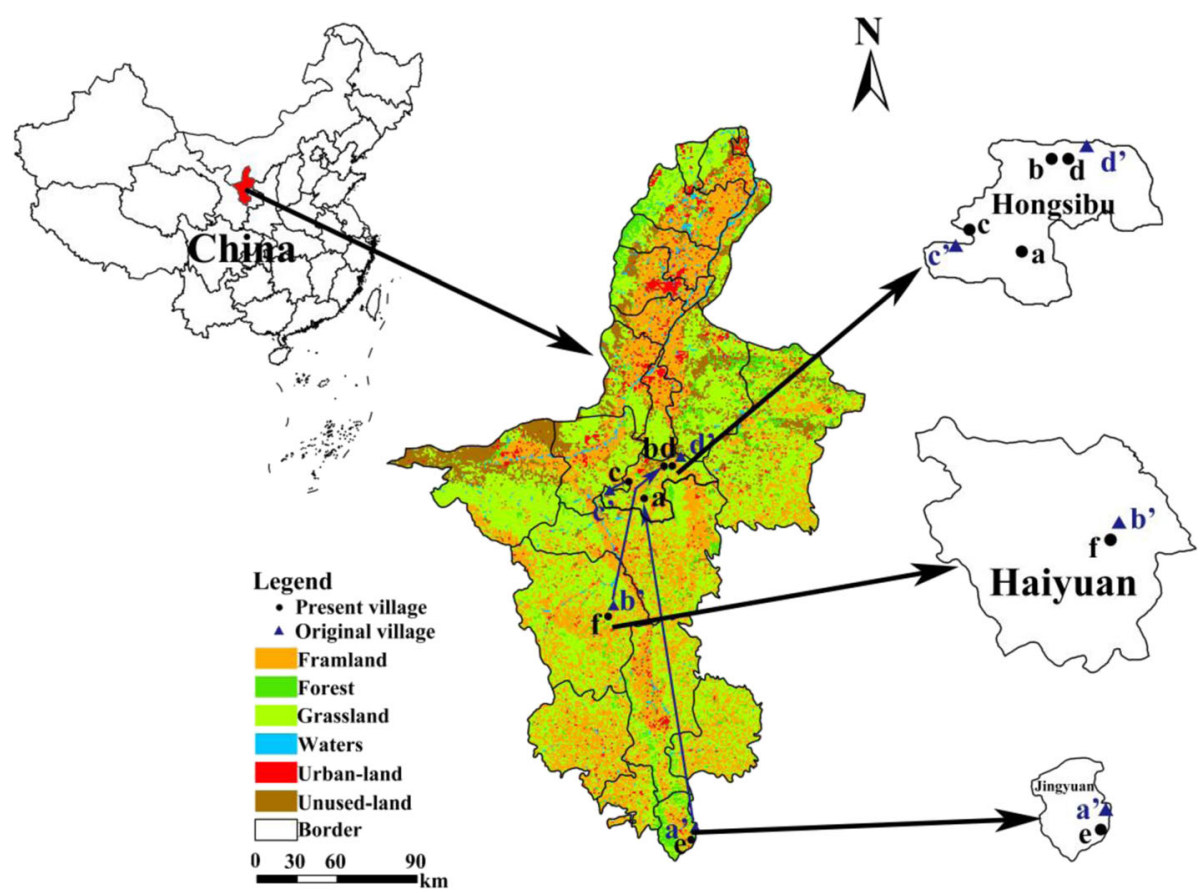

Fig. 1 Distribution map of the surveyed villages

Yellow River to irrigate the barren land. Within 20 years, more than 200,000 people in central and southern Ningxia moved into Hongsibu District, an ecological migration representative area of poverty-alleviation actions. The four villages in the Hongsibu District research area were Liushutai Village Group 2 in Xinzhuang, inhabited by people who moved from Shangwan Village in Xinmin, Jingyuan County, Guyuan, in 2006 (Village a); Yongxin Village Xinquan Group in Liuquan, inhabited by people who moved from Talachuan in Hechuan Village, Jiatang, Haiyuan County, Zhongwei, in 1999 (Village b); Xiangyuan Village Xiangyuan Group in Dahe, inhabited by people who moved from Shuayishan Village in Shitangou Town, Tongxin County, Wuzhong, in 2000 (Village c); and Shuitao Village Group 1 in Liuquan, inhabited by people who moved from Shuitao Village-Wanghutai Village in Weizhou, Tongxin County, Wuzhong, in 2000 (Village d). Among them, Villages $\mathrm{c}$ and $\mathrm{d}$ are semiarid desertified areas, and their immigrants migrated from areas within $5 \mathrm{~km}$.

Jingyuan County of Guyuan $\left(106^{\circ} 12^{\prime}-106^{\circ} 29^{\prime}\right.$ E, $35^{\circ}$ $\left.15^{\prime}-35^{\circ} 37^{\prime} \mathrm{N}\right)$, located in the Liupan Mountain water conservation area in southwestern Ningxia, is the origin of the Jing River, Qingshui River, and Hulu River. It has a relatively humid climate with an annual precipitation of 300-600 mm. This area is covered with diverse types of vegetation, including temperate deciduous broadleaved forests, coniferous and broad-leaved mixed forests, mountain shrub steppe, and alpine and subalpine meadows. Animal and plant species in the area are abundant, offering an important gene pool of animal and plant resources in Ningxia. In this research area was Village e: Zhangtai Village Team 3 in Xinmin, Jingyuan County, Guyuan, which is within a forest area. This village neighbors the location from where the residents of Village a emigrated. Haiyuan County of Zhongwei $\left(105^{\circ} 09^{\prime}-106^{\circ} 10^{\prime} \mathrm{E}, 36^{\circ} 06^{\prime}-37^{\circ} 04^{\prime} \mathrm{N}\right)$ is located in the loess hilly area of Ningxia, which mainly includes loess hilly-gully and soil-rock mixed mountainous areas. It has an annual precipitation of $300-500 \mathrm{~mm}$ and is mainly covered by artificial deciduous broad-leaved forests, forest grasslands, and steppe. In this research area was Village f: Chenwan Team of Baotai Village, Jiatang of Haiyuan County, Zhongwei. It is a loess hilly area that neighbors the location from where the residents of Village b emigrated.

The dominant contributor to the livelihood of the six villages included in this study is animal husbandry, mainly cattle, and sheep farming. Other contributors include crop and forage production, forestry management, and related sectors. The ecological environment of these villages in Ningxia can be divided into three types: semiarid desertified for Villages a, b, c, and d; forest for Village e; and loess hilly for Village f. Correspondingly, the types of forage plant resources vary among the three ecological environment types. Further, considering the migration status, the six villages selected are ideal for assessing changes in traditional forage plant knowledge of ecological migrants. In this study, the impact of ecological migration on the retention of 
Table 1 Geo-relations of six villages in the research area

\begin{tabular}{|c|c|c|c|c|}
\hline Ecological immigrant type & Ecological immigration location & $\begin{array}{l}\text { Migration } \\
\text { distance }\end{array}$ & $\begin{array}{l}\text { Migration } \\
\text { date }\end{array}$ & $\begin{array}{l}\text { Neighboring village of the emigration } \\
\text { area }\end{array}$ \\
\hline $\begin{array}{l}\text { Immigrants from the Liupan } \\
\text { Mountain water conservation } \\
\text { area }\end{array}$ & $\begin{array}{l}\text { Village a, Liushutai Village Group } 2 \text { in } \\
\text { Xinzhuang, inhabited by people who } \\
\text { moved from Shangwan Village in } \\
\text { Xinmin, Jingyuan County, Guyuan }\end{array}$ & $\begin{array}{l}\text { Approximately } \\
300 \mathrm{~km}\end{array}$ & 2006 & $\begin{array}{l}\text { Village e: Zhangtai Village Team } 3 \text { in } \\
\text { Xinmin, Jingyuan County, Guyuan, which } \\
\text { is an unmoved neighboring village of the } \\
\text { original Shangwan Village in Xinmin, } \\
\text { Jingyuan County, Guyuan }\end{array}$ \\
\hline $\begin{array}{l}\text { Immigrants from loess } \\
\text { hilly areas }\end{array}$ & $\begin{array}{l}\text { Village b: Yongxin Village Xinquan Group } \\
\text { in Liuquan, inhabited by people who } \\
\text { moved from Talachuan in Hechuan Village, } \\
\text { Jiatang, Haiyuan County, Zhongwei }\end{array}$ & $\begin{array}{l}\text { Approximately } \\
200 \mathrm{~km}\end{array}$ & 1999 & $\begin{array}{l}\text { Village f: Chenwan Team of Baotai Village } \\
\text { in Jiatang, Haiyuan County, Zhongwei, } \\
\text { which is an unmoved neighboring village } \\
\text { of the original Talachuan natural village of } \\
\text { Hechuan Village in Jiatang, Haiyuan } \\
\text { County, Zhongwei }\end{array}$ \\
\hline $\begin{array}{l}\text { Immigrants from key arid and } \\
\text { sandstorm control areas under } \\
\text { dryland-to-nearby wetland } \\
\text { migration projects }\end{array}$ & $\begin{array}{l}\text { Village c: Xiangyuan Village Xiangyuan } \\
\text { Group in Dahe, inhabited by people who } \\
\text { moved from Shuayishan Village in } \\
\text { Shitangou Town, Tongxin } \\
\text { County, Wuzhong }\end{array}$ & $\begin{array}{l}\text { Approximately } \\
20 \mathrm{~km}\end{array}$ & 2000 & No residents near the emigration area \\
\hline $\begin{array}{l}\text { Immigrants from key arid and } \\
\text { sandstorm control areas under } \\
\text { dryland-to-nearby wetland } \\
\text { migration projects }\end{array}$ & $\begin{array}{l}\text { Village d: Shuitao Village Group } 1 \text { in } \\
\text { Liuquan, inhabited by people who moved } \\
\text { from Shuitao Village - Wanghutai Village in } \\
\text { Weizhou, Tongxin County, Wuzhong }\end{array}$ & $\begin{array}{l}\text { Approximately } \\
2 \mathrm{~km}\end{array}$ & 2000 & No residents near the emigration area \\
\hline
\end{tabular}

The dryland-to-nearby wetland migration projects resettle immigrants from deep mountain areas lacking water sources and transportation to nearby flat areas with water sources, with the migration distance less than $20 \mathrm{~km}$

Neighboring village of the emigration area refers to the village that remains in the vicinity of the immigrants' original residence

traditional forage plant knowledge was analyzed through evaluating the similarities and differences between migrants and residents in the unmoved village neighboring the original emigration location (Tables 1 and 2).

\section{Data collection}

Data were collected from March 2018 to May 2019. During the initial stage from March to May 2018, a preliminary investigation of ecological migration projects and forage plant resources in Ningxia was conducted, from which six representative villages were selected for data collection. Subsequently, field surveys were conducted in the six villages from June 2018 to May 2019. With the help of general secretaries of local village committees, heads of forest and grassland stations, and local livestock farming masters ("Yang ba shi"), 315 participants (165 males and 150 females) were sampled through a snowballing technique and given semistructured interviews. The interviewed households accounted for approximately $30 \%$ of total households in each village $[7,9,28]$. Prior to the study, informed consent was obtained from all participants regarding the interview procedures. The demographic characteristics, including gender, age, education level, and occupation, are presented in Table $3[4,8]$.

In the field surveys, respondents were invited to freely list the forage plants they used $[8,29]$. The interview included the following questions: (1) Do you know which plants cattle and sheep like to eat? (2) What plants do you feed your cattle and sheep? (3) What parts of these plants do cattle and sheep eat? (4) Are these plants fed to cattle and sheep as tender grass or stored green hay? (5) What types of plants are used to feed livestock in each season? (6) Is there any difference in the plants fed to cattle and sheep before and after migration?

In the second stage of the field study, accompanied by plant experts from the local forest and grassland station and by livestock farming masters, the investigators identified and collected forage plant samples and documented the detailed information of each sample. Voucher specimens and physical samples of forage plants were collected according to various identification documents, including

Table 2 Basic information of the six villages in the research area

\begin{tabular}{|c|c|c|c|c|c|c|}
\hline The village & Number of households & Population & Number of households interviewed & Interview proportion & Altitude (m) & Geographic location \\
\hline Village a & 118 & 560 & 42 & $36 \%$ & 1475 & $106^{\circ} 10^{\prime} \mathrm{E}, 37^{\circ} 27^{\prime} \mathrm{N}$ \\
\hline Village $b$ & 101 & 370 & 39 & $39 \%$ & 1300 & $106^{\circ} 24^{\prime} \mathrm{E}, 37^{\circ} 46^{\prime} \mathrm{N}$ \\
\hline Village c & 240 & 820 & 73 & $30 \%$ & 1300 & $105^{\circ} 99^{\prime} \mathrm{E}, 37^{\circ} 37^{\prime} \mathrm{N}$ \\
\hline Village d & 180 & 730 & 68 & $38 \%$ & 1263 & $106^{\circ} 29^{\prime} \mathrm{E}, 37^{\circ} 46^{\prime} \mathrm{N}$ \\
\hline Village e & 128 & 538 & 48 & $38 \%$ & 1821 & $106^{\circ} 47^{\prime} \mathrm{E}, 35^{\circ} 36^{\prime} \mathrm{N}$ \\
\hline Village $f$ & 120 & 397 & 45 & $38 \%$ & 1593 & $105^{\circ} 86^{\prime} \mathrm{E}, 36^{\circ} 60^{\prime} \mathrm{N}$ \\
\hline
\end{tabular}


Flora of China, Flora of Ningxia, Flora of Liupan Mountain, Atlas of Animals and Plants of Nanhua Mountain in Ningxia, and Vascular Plants in Luoshan Mountain of Ningxia. All voucher specimens were stored in the College of Life and Environmental Sciences, Minzu University of China. After collection and preliminary identification, the plant samples were identified and verified by plant taxonomic experts, including Qiang Zhu from the Ningxia Forestry Research Institute, Bo Liu from Minzu University of China, Sheng Qiang from Nanjing Agricultural University, and Kechang Li, a former head of a forest and grassland station in Ningxia.

\section{Data analysis}

Traditional forage plant information collected from 315 respondents was analyzed using four data analysis methods.

\section{Relative frequency of citation (RFC): $R F C=\frac{F C s}{N}$}

This parameter refers to the ratio of the number of respondents who mention a particular forage plant (i.e., frequency of citation, FC) to the number of all respondents participating in the survey $(N)$. The larger the RFC, the more important and valuable the forage plant is in the area. The importance of each forage plant was indicated by its FC value, which allowed all forage plants mentioned in the survey to be listed in order of importance $[30,31]$.

\section{Jaccard $J I=\frac{C}{A+b-C} \times 100$ index (JI):}

where $A$ represents the number of forage plant species used by villagers in Village a, $B$ represents the number of forage plant species used by villagers in Village $b$, and $C$ represents the number of forage plant species used by both Village a and Village b. The JI can be used to compare and evaluate the similarity in the use of forage plant species between two villages [32]. The JI value is between 0 and 100. The greater the value is, the more similar two villages are in using forage plants; low values indicate that there is a great difference in the understanding and utilization of plants between the two villages [33, 34].

\section{Cluster analysis}

The types of forage plants used by the villagers were recorded and counted for analysis with each village as a unit. Using the chi-square value of the number of forage plant types used in each village as a distance function, systematic cluster analysis was performed to evaluate the similarity in the use of forage plants between villages by using an intergroup classification method. The greater the similarity is, the smaller the distance function. Subsequently, a classification was conducted based on distance function. Cluster analysis was used as a supplement to the JI-based assessment to verify the similarities and differences in the diversity of traditional forage plants used by villagers from different villages.

\section{Multivariate analysis of variance (MANOVA)}

Four factors (gender, age, education level, and identity/occupation of the respondents) were used as reference variables. MANOVA was performed to evaluate whether the four reference variables had a significant impact on the number of forage plants mentioned by the respondents.

\section{Results}

Diversity and distribution of traditional forage plants

A total of 224 forage plant species belonging to 150 genera in 42 families were enumerated by respondents during the survey (Table 4). The following are the plant families that included more than ten traditional forage plant species: Leguminosae (33 species, 14.10\%), Compositae (29 species, 12.39\%), Gramineae (24 species,

Table 3 Sociodemographic characteristics of participants

\begin{tabular}{|c|c|c|c|c|c|c|c|c|c|c|c|c|c|c|c|c|c|}
\hline \multirow[t]{2}{*}{ Category } & \multicolumn{2}{|c|}{ Gender } & \multicolumn{4}{|l|}{ Age } & \multicolumn{4}{|c|}{ Education Level } & \multicolumn{7}{|c|}{ Occupation } \\
\hline & Male & Female & $\leq 19$ & 20-39 & $40-59$ & $\geq 60$ & 1 & $P$ & $M$ & $\mathrm{H}$ & V & $\mathrm{F}$ & B & W & $\mathrm{D}$ & $S$ & $\mathrm{O}$ \\
\hline Village a & 21 & 21 & 4 & 10 & 18 & 10 & 13 & 22 & 4 & 3 & 3 & 32 & 0 & 1 & 1 & 4 & 1 \\
\hline Village b & 21 & 18 & 4 & 13 & 17 & 5 & 16 & 16 & 4 & 3 & 1 & 27 & 1 & 6 & 0 & 3 & 1 \\
\hline Village c & 42 & 31 & 5 & 13 & 38 & 17 & 27 & 32 & 11 & 3 & 3 & 61 & 0 & 2 & 1 & 5 & 1 \\
\hline Village $d$ & 27 & 41 & 6 & 24 & 21 & 17 & 31 & 19 & 16 & 2 & 2 & 57 & 2 & 3 & 0 & 3 & 1 \\
\hline Village e & 26 & 22 & 6 & 11 & 19 & 12 & 18 & 16 & 9 & 5 & 1 & 31 & 2 & 4 & 3 & 5 & 2 \\
\hline Village $f$ & 28 & 17 & 3 & 14 & 14 & 14 & 23 & 14 & 5 & 3 & 2 & 36 & 0 & 3 & 0 & 3 & 1 \\
\hline Quantity & 165 & 150 & 28 & 85 & 127 & 75 & 128 & 119 & 49 & 19 & 12 & 244 & 5 & 19 & 5 & 23 & 7 \\
\hline Proportion & $52 \%$ & $48 \%$ & $9 \%$ & $27 \%$ & $40 \%$ & $24 \%$ & $40 \%$ & $38 \%$ & $16 \%$ & $6 \%$ & $4 \%$ & $77 \%$ & $2 \%$ & $6 \%$ & $2 \%$ & $7 \%$ & $2 \%$ \\
\hline
\end{tabular}

Education level: I = Illiteracy; $\mathrm{P}=$ primary school; $\mathrm{M}=$ middle high school; $\mathrm{H}=$ High school. Occupation: $\mathrm{V}=$ village heads; $\mathrm{F}=\mathrm{farmers} ; \mathrm{B}=$ businessmen; $\mathrm{W}=$ migrant workers; $\mathrm{D}=$ village doctors; $\mathrm{S}=$ students; $\mathrm{O}=$ others 
10.26\%), Amaranthaceae (21 species, 8.97\%), Rosaceae (15 species, 6.41\%), and Polygonaceae (12 species, 5.13\%) (Fig. 2). Among 224 forage plant species, 181 (81\%) are herbaceous and lianoid plants, and 43 (19\%) are trees and shrubs, suggesting that the local farmers and herdsmen mainly depend on herbaceous plants as livestock feed. For most traditional forage plants (213 species, 95\%), stems and leaves are the most common parts used for livestock feed; among them, for 39 species (17.4\%), flowers and fruit were also used for livestock feed. This indicates a high diversity of local forage plant resources. For 11 species $(0.05 \%)$, only roots, flowers, and seeds can be used for livestock feed. Although these forage plants only account for a small proportion, each has its own unique characteristics and should not be ignored. For example, in two plant species (0.008\%), Potentilla anserina and Daucus Carota, roots are the only part used for livestock feed, but they are highly favored by cattle and sheep due to a rich starch content and juicy taste. In three species (0.013\%), Schisandra chinensis, Sophora alopecuroides, and Eruca vesicaria, only the fruit is used for livestock feed. The fruit of S. chinensis, which has a sour, "numbing," and spicy taste, can be used as medicine. For S. alopecuroides, only the seeds can be fed to sheep and cattle, but they are seasonally toxic, thus requiring more caution when used as feed. E. vesicaria is a local oil crop, and its oil cake and oil residue are nutritious livestock feed. For two plant species (0.008\%), Artemisia argyi and Ammopiptanthus mongolicus, only the flowers can be used for livestock feed. The former is mainly used for medicinal moxibustion in local areas, but its inflorescence is occasionally used to feed cattle and sheep. For A. mongolicus, only sheep eat some flowers.

\section{Value and knowledge of major forage plants for feeding livestock}

The RFC value calculated in this study ranged from 0.32 to 77.14. For each forage plant species, the higher the RFC value, the more frequently it is used by local villagers to feed livestock, and the more important and valuable it is in the area. The top 13 plant species with an RFC value greater than 50 were Agropyron cristatum (RFC 77.1), Artemisia scoparia (RFC 73.0), Salsola collina (RFC 71.4), Chenopodium album (RFC 69.2), Medicago ruthenica (RFC 67.9), Artemisia capillaris (RFC 67.6), Setaria viridis (RFC 67.3), Chenopodium glaucum (RFC 65.1), Zea mays (RFC 56.8), Sonchus (RFC 56.2), Sonchus oleraceus (RFC 56.2), Medicago falcata (RFC 55.9), and Echinochloa crus-galli (RFC 54.9). These plants have a good palatability and are favorite feed of cattle and sheep. Their stems and leaves are the common parts used for livestock feed in the form of either tender grass or green hay. Among them, maize (Z. mays) and alfalfa ( $M$. ruthenica) are cultivated forage grasses with high forage value. Wheatgrass (A. cristatum), green bristlegrass (S. viridis), and paspalum (E. crus-galli) have a massive amount of leaves and soft stems favored by cattle and sheep. M. falcata is favored by domestic livestock during the entire plant growth period and after withering; green and tender plants can increase the milk yield of dairy livestock, stems left after defoliation in winter are favorite forage of livestock, and the green hay of this species is also good winter forage. For C. glaucum, despite its high RFC value as forage for both cattle and sheep in the forms of either green tender plants or dry hay, the villagers warned that the plants can cause itching or swelling of the whole body if livestock consumes too much.

$S$. arvensis, $S$. oleraceus, and $L$. tatarica are three important local forage plants with RFC values of 56.2, 56.2, and 52.7, respectively. These three forage plant species, which were mentioned by all the respondents from Village f, share a common Chinese name, Kukucai, because of their bitter taste. They are edible and medicinal and can be used for livestock feed. Kukucai is commonly used in a homemade local cold dish. When used as an anti-inflammatory medicine, it is commonly referred to as Patrinia villosa. In addition, the juicy stems and leaves of Kukucai are favorite forage of cattle and sheep. These applications fully reflect the important value of Kukucai.

\section{Comparison of traditional forage plants in six villages in the study area}

Among the 224 identified plants, 40 species (18\%) were mentioned in Village a, 52 species $(23 \%)$ in Village b, 132 species (59\%) in Village c, 123 species (55\%) in Village d, 121 species (54\%) in Village e, and 118 species $(53 \%)$ in Village $\mathrm{f}$. In terms of the number of forage plant species reported, Village $c>$ Village $d>$ Village e $>$ Village $\mathrm{f}>$ Village $\mathrm{b}>$ Village a. Villagers in Villages a and $\mathrm{b}$ reported fewer forage plant species, only approximately $20 \%$ of the total. In contrast, Villages c, d, e, and f each mentioned more than 100 forage plant species, approximately $50 \%$ of the total forage plant species reported. The number of forage plant species used by the villagers in Villages a and b was significantly lower, by $33 \%$ and $44 \%$, respectively, than the number of those used by the villagers in Villages e and $f$ near their original living areas. Long-distance migration brought prominent changes in the ecological environment and natural resources to the villagers of Villages a and b. In particular, for Village a, which moved from a forest area to a semiarid desertified area, the number of traditional forage plant species used by villagers showed a significant decreasing trend, indicating that the relocation had 
Table 4 Traditional forage plants and their relative frequency of citation (RFC)

\begin{tabular}{|c|c|c|c|c|c|c|c|c|c|c|c|c|c|}
\hline \multirow[t]{2}{*}{ Family name } & \multirow[t]{2}{*}{ Scientific name } & \multirow[t]{2}{*}{$\begin{array}{l}\text { Chinese } \\
\text { name }\end{array}$} & \multirow[t]{2}{*}{ Local name } & \multirow[t]{2}{*}{$\begin{array}{l}\text { Life } \\
\text { form }\end{array}$} & \multirow[t]{2}{*}{$\begin{array}{l}\text { Part } \\
\text { used }\end{array}$} & \multicolumn{6}{|c|}{$\begin{array}{l}\text { Frequency of citations } \\
\text { in the surveyed villages }\end{array}$} & \multirow{2}{*}{$\begin{array}{l}\text { Frequency } \\
\text { of citation (FC) }\end{array}$} & \multirow{2}{*}{$\begin{array}{l}\text { Relative } \\
\text { frequency } \\
\text { of citation } \\
\text { (RFC) }\end{array}$} \\
\hline & & & & & & $a$ & b & c & $d$ & e & $f$ & & \\
\hline Schisandraceae & $\begin{array}{l}\text { Schisandra } \\
\text { chinensis } \\
\text { (Turcz.) Baill. }\end{array}$ & $\begin{array}{l}\text { Wu Wei } \\
\text { Zi }\end{array}$ & $\begin{array}{l}\text { Chan tiao wan, Ye pu } \\
\text { tao wan }\end{array}$ & Liana & Fruit & 0 & 0 & 0 & 0 & 2 & 0 & 2 & 0.63 \\
\hline Xanthorrhoeaceae & $\begin{array}{l}\text { Hemerocallis citrina } \\
\text { Baroni }\end{array}$ & $\begin{array}{l}\text { Huang } \\
\text { Hua Cai }\end{array}$ & $\begin{array}{l}\text { Huang hua cai, Jin } \\
\text { zhen hua }\end{array}$ & Herb & $\begin{array}{l}\text { Stem, } \\
\text { leaf }\end{array}$ & 0 & 0 & 32 & 0 & 0 & 0 & 32 & 10.16 \\
\hline Amaryllidaceae & $\begin{array}{l}\text { Allium mongolicum } \\
\text { Regel }\end{array}$ & $\begin{array}{l}\text { Meng } \\
\text { Gu Jiu }\end{array}$ & Sha cong & Herb & $\begin{array}{l}\text { Stem, } \\
\text { leaf }\end{array}$ & 0 & 0 & 53 & 15 & 0 & 1 & 69 & 21.9 \\
\hline Amaryllidaceae & $\begin{array}{l}\text { Allium polyrhizum } \\
\text { Turcz. ex Regel }\end{array}$ & Jian Jiu & Shi cong & Herb & $\begin{array}{l}\text { Stem, } \\
\text { leaf }\end{array}$ & 0 & 0 & 25 & 15 & 0 & 0 & 40 & 12.7 \\
\hline Amaryllidaceae & $\begin{array}{l}\text { Allium tenuissimum } \\
\mathrm{L} \text {. }\end{array}$ & Xi Ye Jiu & Yang hu zi & Herb & $\begin{array}{l}\text { Stem, } \\
\text { leaf }\end{array}$ & 0 & 0 & 0 & 0 & 0 & 28 & 28 & 8.89 \\
\hline Typhaceae & $\begin{array}{l}\text { Typha domingensis } \\
\text { Pers. }\end{array}$ & $\begin{array}{l}\text { Chang } \\
\text { Bao } \\
\text { Xiang } \\
\text { Pu }\end{array}$ & Mao la & Herb & $\begin{array}{l}\text { Stem, } \\
\text { leaf }\end{array}$ & 0 & 0 & 0 & 0 & 0 & 6 & 6 & 1.9 \\
\hline Poaceae & $\begin{array}{l}\text { Agropyron } \\
\text { cristatum }\end{array}$ & $\begin{array}{l}\text { Bing } \\
\text { Cao }\end{array}$ & $\begin{array}{l}\text { Bing cao, Bi dang } \\
\text { zi, Huang shu yi ba }\end{array}$ & Herb & $\begin{array}{l}\text { Stem, } \\
\text { leaf }\end{array}$ & 35 & 23 & 57 & 54 & 36 & 38 & 243 & 77.14 \\
\hline Poaceae & Avena sativa $\mathrm{L}$. & Yan Mai & $\begin{array}{l}\text { Da yan mai, Da yan, } \\
\text { Huo yan mai }\end{array}$ & Herb & $\begin{array}{l}\text { Stem, } \\
\text { leaf }\end{array}$ & 2 & 0 & 0 & 0 & 33 & 26 & 61 & 19.37 \\
\hline Poaceae & $\begin{array}{l}\text { Calamagrostis } \\
\text { epigejos (L.) Roth. }\end{array}$ & $\begin{array}{l}\text { Fu Zi } \\
\text { Mao }\end{array}$ & Lu cao & Herb & $\begin{array}{l}\text { Stem, } \\
\text { leaf }\end{array}$ & 0 & 0 & 3 & 39 & 0 & 30 & 72 & 22.86 \\
\hline Poaceae & Chloris virgata Sw. & $\begin{array}{l}\text { Hu Wei } \\
\text { Cao }\end{array}$ & $\begin{array}{l}\text { Gou wei cao, Ba } \\
\text { ban cao }\end{array}$ & Herb & $\begin{array}{l}\text { Stem, } \\
\text { leaf }\end{array}$ & 3 & 4 & 20 & 1 & 0 & 0 & 28 & 8.89 \\
\hline Poaceae & $\begin{array}{l}\text { Cleistogenes } \\
\text { squarrosa (Trin.) } \\
\text { Keng }\end{array}$ & $\begin{array}{l}\text { Zao Yin } \\
\text { Zi Cao }\end{array}$ & Xuan feng cao & Herb & $\begin{array}{l}\text { Stem, } \\
\text { leaf }\end{array}$ & 0 & 7 & 45 & 34 & 0 & 23 & 109 & 34.6 \\
\hline Poaceae & $\begin{array}{l}\text { Echinochloa crus- } \\
\text { galli (L.) P.Beauv. }\end{array}$ & Bai & $\begin{array}{l}\text { Bai zi cao, } \\
\text { Bing cao }\end{array}$ & Herb & $\begin{array}{l}\text { Stem, } \\
\text { leaf, } \\
\text { seed }\end{array}$ & 35 & 12 & 3 & 49 & 36 & 38 & 173 & 54.92 \\
\hline Poaceae & $\begin{array}{l}\text { Eragrostis } \\
\text { ferruginea (Thunb.) } \\
\text { P.Beauv. }\end{array}$ & $\begin{array}{l}\text { Xiao } \\
\text { Hua Mei } \\
\text { Cao }\end{array}$ & $\begin{array}{l}\text { Xiang mao zi, } \\
\text { Xiang mao }\end{array}$ & Herb & $\begin{array}{l}\text { Stem, } \\
\text { leaf }\end{array}$ & 0 & 4 & 59 & 52 & 0 & 30 & 145 & 46.03 \\
\hline Poaceae & $\begin{array}{l}\text { Koeleria } \\
\text { pyramidata (Lam.) } \\
\text { P.Beauv. }\end{array}$ & Da Cao & Bing cao, Liu yue he & Herb & $\begin{array}{l}\text { Stem, } \\
\text { leaf }\end{array}$ & 0 & 0 & 0 & 0 & 36 & 0 & 36 & 11.43 \\
\hline Poaceae & $\begin{array}{l}\text { Leymus secalinus } \\
\text { (Georgi) Tzvelev }\end{array}$ & Lai Cao & Bing cao & Herb & $\begin{array}{l}\text { Stem, } \\
\text { leaf }\end{array}$ & 0 & 0 & 0 & 0 & 0 & 38 & 38 & 12.06 \\
\hline Poaceae & $\begin{array}{l}\text { Panicum miliaceum } \\
\mathrm{L} \text {. }\end{array}$ & $\mathrm{Mi} \mathrm{Zi}$ & $\begin{array}{l}\text { Mi zi cao, Hong mi zi, } \\
\text { Huang mi zi }\end{array}$ & Herb & $\begin{array}{l}\text { Stem, } \\
\text { leaf }\end{array}$ & 0 & 0 & 0 & 16 & 0 & 41 & 57 & 18.1 \\
\hline Poaceae & $\begin{array}{l}\text { Pennisetum } \\
\text { centrasiaticum } \\
\text { Tzvelev }\end{array}$ & $\begin{array}{l}\text { Zhong } \\
\text { Ya Bai } \\
\text { Cao }\end{array}$ & $\begin{array}{l}\text { bia cao, Lang wei cao, } \\
\text { Dao sheng zi }\end{array}$ & Herb & $\begin{array}{l}\text { Stem, } \\
\text { leaf }\end{array}$ & 0 & 9 & 3 & 18 & 0 & 30 & 60 & 19.05 \\
\hline Poaceae & $\begin{array}{l}\text { Phragmites } \\
\text { australis (Cav.) Trin. } \\
\text { ex Steud. }\end{array}$ & Lu Wei & Lu nia, Lu cao & Herb & $\begin{array}{l}\text { Stem, } \\
\text { leaf }\end{array}$ & 0 & 0 & 0 & 0 & 19 & 0 & 19 & 6.03 \\
\hline Poaceae & Secale cereale L. & Hei Mai & Ye yang mai & Herb & $\begin{array}{l}\text { Stem, } \\
\text { leaf }\end{array}$ & 0 & 0 & 0 & 0 & 31 & 0 & 31 & 9.84 \\
\hline Poaceae & $\begin{array}{l}\text { Setaria italica (L.) } \\
\text { P.Beauv. }\end{array}$ & Gu Zi & He cao, Gu cao & Herb & $\begin{array}{l}\text { Stem, } \\
\text { leaf }\end{array}$ & 0 & 0 & 0 & 0 & 0 & 35 & 35 & 11.11 \\
\hline Poaceae & $\begin{array}{l}\text { Setaria viridis (L.) } \\
\text { P.Beauv. }\end{array}$ & $\begin{array}{l}\text { Gou } \\
\text { Wei Cao }\end{array}$ & $\begin{array}{l}\text { Gu you zi, } \\
\text { Mao er gu zhu }\end{array}$ & Herb & $\begin{array}{l}\text { Stem, } \\
\text { leaf }\end{array}$ & 5 & 15 & 63 & 59 & 35 & 35 & 212 & 67.3 \\
\hline Poaceae & $\begin{array}{l}\text { Sorghum bicolor } \\
\text { (L.) Moench }\end{array}$ & $\begin{array}{l}\text { Gao } \\
\text { Liang }\end{array}$ & $\begin{array}{l}\text { Gao liang, } \\
\text { Chu chu }\end{array}$ & Herb & $\begin{array}{l}\text { Stem, } \\
\text { leaf }\end{array}$ & 0 & 1 & 0 & 0 & 27 & 17 & 45 & 14.29 \\
\hline
\end{tabular}


Table 4 Traditional forage plants and their relative frequency of citation (RFC) (Continued)

\begin{tabular}{|c|c|c|c|c|c|c|c|c|c|c|c|c|c|}
\hline \multirow[t]{2}{*}{ Family name } & \multirow[t]{2}{*}{ Scientific name } & \multirow[t]{2}{*}{$\begin{array}{l}\text { Chinese } \\
\text { name }\end{array}$} & \multirow[t]{2}{*}{ Local name } & \multirow[t]{2}{*}{$\begin{array}{l}\text { Life } \\
\text { form }\end{array}$} & \multirow[t]{2}{*}{$\begin{array}{l}\text { Part } \\
\text { used }\end{array}$} & \multicolumn{6}{|c|}{$\begin{array}{l}\text { Frequency of citations } \\
\text { in the surveyed villages }\end{array}$} & \multirow{2}{*}{$\begin{array}{l}\text { Frequency } \\
\text { of citation (FC) }\end{array}$} & \multirow{2}{*}{$\begin{array}{l}\text { Relative } \\
\text { frequency } \\
\text { of citation } \\
\text { (RFC) }\end{array}$} \\
\hline & & & & & & a & $b$ & c & $d$ & e & $f$ & & \\
\hline Poaceae & $\begin{array}{l}\text { Stipa tianschanica } \\
\text { Roshev. }\end{array}$ & $\begin{array}{l}\text { Ge Bi } \\
\text { Zhen } \\
\text { Mao }\end{array}$ & Suo cao & Herb & $\begin{array}{l}\text { Stem, } \\
\text { leaf }\end{array}$ & 0 & 0 & 53 & 58 & 0 & 36 & 147 & 46.67 \\
\hline Poaceae & $\begin{array}{l}\text { Stipa breviflora } \\
\text { Griseb. }\end{array}$ & $\begin{array}{l}\text { Duan } \\
\text { Hua } \\
\text { Zhen } \\
\text { Mao }\end{array}$ & Suo cao & Herb & $\begin{array}{l}\text { Stem, } \\
\text { leaf }\end{array}$ & 0 & 0 & 53 & 58 & 0 & 36 & 147 & 46.67 \\
\hline Poaceae & $\begin{array}{l}\text { Stipa bungeana } \\
\text { Trin. }\end{array}$ & $\begin{array}{l}\text { Chang } \\
\text { Mang } \\
\text { Cao }\end{array}$ & Suo cao & Herb & $\begin{array}{l}\text { Stem, } \\
\text { leaf }\end{array}$ & 0 & 0 & 53 & 58 & 0 & 36 & 147 & 46.67 \\
\hline Poaceae & $\begin{array}{l}\text { Stipa caucasica } \\
\text { Schmalh. }\end{array}$ & $\begin{array}{l}\text { Sha } \\
\text { Sheng } \\
\text { Zhen } \\
\text { Mao }\end{array}$ & Suo cao & Herb & $\begin{array}{l}\text { Stem, } \\
\text { leaf }\end{array}$ & 0 & 0 & 53 & 58 & 0 & 36 & 147 & 46.67 \\
\hline Poaceae & $\begin{array}{l}\text { Stipa grandis } \\
\text { P.A.Smirn. }\end{array}$ & $\begin{array}{l}\text { Da } \\
\text { Zhen } \\
\text { Mao }\end{array}$ & Suo cao & Herb & $\begin{array}{l}\text { Stem, } \\
\text { leaf }\end{array}$ & 0 & 0 & 53 & 58 & 0 & 36 & 147 & 46.67 \\
\hline Poaceae & $\begin{array}{l}\text { Stipa splendens } \\
\text { Trin. }\end{array}$ & Ji Ji Cao & Ji ji, Xi ji hu zi, Suan cao & Herb & $\begin{array}{l}\text { Stem, } \\
\text { leaf }\end{array}$ & 2 & 6 & 4 & 6 & 20 & 39 & 77 & 24.44 \\
\hline Poaceae & $\begin{array}{l}\text { Triticum aestivum } \\
\mathrm{L} .\end{array}$ & $\begin{array}{l}\text { Xiao } \\
\text { Mai }\end{array}$ & $\begin{array}{l}\text { Hong mang dong mai, } \\
\text { Mia cao, Hong mang } \\
\text { chun mai }\end{array}$ & Herb & $\begin{array}{l}\text { Stem, } \\
\text { leaf }\end{array}$ & 0 & 0 & 33 & 0 & 0 & 35 & 68 & 21.59 \\
\hline Poaceae & Zea mays $\mathrm{L}$. & Yu Mi & Yu mi & Herb & $\begin{array}{l}\text { Stem, } \\
\text { leaf }\end{array}$ & 12 & 18 & 47 & 45 & 48 & 9 & 179 & 56.83 \\
\hline Berberidaceae & $\begin{array}{l}\text { Berberis } \\
\text { brachypoda } \\
\text { Maxim. }\end{array}$ & $\begin{array}{l}\text { Duan } \\
\text { Bing } \\
\text { Xiao Bo }\end{array}$ & Suan bu liu shu & Shrub & Leaf & 0 & 0 & 0 & 0 & 8 & 0 & 8 & 2.54 \\
\hline Ranunculaceae & $\begin{array}{l}\text { Actaea asiatica } \\
\text { H.Hara }\end{array}$ & $\begin{array}{l}\text { Lei Ye } \\
\text { Sheng } \\
\text { Ma }\end{array}$ & Mi la de gan & Herb & $\begin{array}{l}\text { Stem, } \\
\text { leaf }\end{array}$ & 0 & 0 & 0 & 0 & 10 & 0 & 10 & 3.17 \\
\hline Ranunculaceae & $\begin{array}{l}\text { Actaea cimicifuga } \\
\text { L. }\end{array}$ & $\begin{array}{l}\text { Sheng } \\
\text { Ma }\end{array}$ & Sheng ma & Herb & $\begin{array}{l}\text { Stem, } \\
\text { leaf }\end{array}$ & 0 & 0 & 0 & 0 & 5 & 0 & 5 & 1.59 \\
\hline Ranunculaceae & $\begin{array}{l}\text { Thalictrum } \\
\text { petaloideum L. }\end{array}$ & $\begin{array}{l}\text { Ban Rui } \\
\text { Tang } \\
\text { Song } \\
\text { Cao }\end{array}$ & $\begin{array}{l}\text { Nai de cao, Yang nai cao, } \\
\text { Yang nai de hua }\end{array}$ & Herb & Leaf & 0 & 0 & 0 & 0 & 22 & 0 & 22 & 6.98 \\
\hline Grossulariaceae & $\begin{array}{l}\text { Ribes } \\
\text { maximowiczianum } \\
\text { Komarov }\end{array}$ & $\begin{array}{l}\text { Jian Ye } \\
\text { Cha } \\
\text { Biao Zi }\end{array}$ & Cha ye mu & Shrub & Leaf & 0 & 0 & 0 & 0 & 3 & 0 & 3 & 0.95 \\
\hline Zygophyllaceae & Tribulus terrestris L. & $\mathrm{Ji} \mathrm{Li}$ & Ba jue zi & Herb & $\begin{array}{l}\text { Stem, } \\
\text { leaf }\end{array}$ & 5 & 3 & 63 & 57 & 0 & 30 & 158 & 50.16 \\
\hline Leguminosae & $\begin{array}{l}\text { Ammopiptanthus } \\
\text { mongolicus (Kom.) } \\
\text { S.H.Cheng }\end{array}$ & $\begin{array}{l}\text { Sha } \\
\text { Dong } \\
\text { Qing }\end{array}$ & Dong qing & Shrub & Flower & 0 & 0 & 8 & 0 & 0 & 0 & 8 & 2.54 \\
\hline Leguminosae & $\begin{array}{l}\text { Astragalus } \\
\text { efoliolatus Hand.- } \\
\text { Mazz. }\end{array}$ & $\begin{array}{l}\text { Dan Ye } \\
\text { Huang } \\
\text { Qi }\end{array}$ & Mao ti ti hua & Herb & $\begin{array}{l}\text { Stem, } \\
\text { leaf }\end{array}$ & 0 & 0 & 4 & 8 & 0 & 3 & 15 & 4.76 \\
\hline Leguminosae & $\begin{array}{l}\text { Astragalus } \\
\text { scaberrimus Bunge }\end{array}$ & $\begin{array}{l}\text { Cao Ye } \\
\text { Huang } \\
\text { Qi }\end{array}$ & Mao ti ti hua & Herb & $\begin{array}{l}\text { Stem, } \\
\text { leaf }\end{array}$ & 0 & 0 & 0 & 0 & 0 & 3 & 3 & 0.95 \\
\hline Leguminosae & $\begin{array}{l}\text { Astragalus } \\
\text { propinquus } \\
\text { Schischkin }\end{array}$ & $\begin{array}{l}\text { Huang } \\
\text { Qi }\end{array}$ & Huang qi & Herb & $\begin{array}{l}\text { Stem, } \\
\text { leaf }\end{array}$ & 0 & 0 & 0 & 0 & 3 & 2 & 5 & 1.59 \\
\hline Leguminosae & $\begin{array}{l}\text { Caragana } \\
\text { korshinskii Kom. }\end{array}$ & $\begin{array}{l}\text { Ning } \\
\text { Tiao Jin }\end{array}$ & Niu ban jing cao & Shrub & $\begin{array}{l}\text { Stem, } \\
\text { leaf }\end{array}$ & 11 & 2 & 11 & 37 & 0 & 28 & 89 & 28.25 \\
\hline
\end{tabular}


Table 4 Traditional forage plants and their relative frequency of citation (RFC) (Continued)

\begin{tabular}{|c|c|c|c|c|c|c|c|c|c|c|c|c|c|}
\hline \multirow[t]{2}{*}{ Family name } & \multirow[t]{2}{*}{ Scientific name } & \multirow[t]{2}{*}{$\begin{array}{l}\text { Chinese } \\
\text { name }\end{array}$} & \multirow[t]{2}{*}{ Local name } & \multirow[t]{2}{*}{$\begin{array}{l}\text { Life } \\
\text { form }\end{array}$} & \multirow[t]{2}{*}{$\begin{array}{l}\text { Part } \\
\text { used }\end{array}$} & \multicolumn{6}{|c|}{$\begin{array}{l}\text { Frequency of citations } \\
\text { in the surveyed villages }\end{array}$} & \multirow[t]{2}{*}{$\begin{array}{l}\text { Frequency } \\
\text { of citation (FC) }\end{array}$} & \multirow{2}{*}{$\begin{array}{l}\text { Relative } \\
\text { frequency } \\
\text { of citation } \\
\text { (RFC) }\end{array}$} \\
\hline & & & & & & a & b & c & $d$ & e & f & & \\
\hline & & Ji Er & & & & & & & & & & & \\
\hline Leguminosae & $\begin{array}{l}\text { Caragana } \\
\text { stenophylla Pojark. }\end{array}$ & $\begin{array}{l}\text { Xia Ye } \\
\text { Jin Ji Er }\end{array}$ & Niu ban jing ci & Shrub & $\begin{array}{l}\text { Stem, } \\
\text { flower, } \\
\text { fruit }\end{array}$ & 0 & 0 & 3 & 2 & 0 & 28 & 33 & 10.48 \\
\hline Leguminosae & $\begin{array}{l}\text { Caragana tibetica } \\
\text { Kom. }\end{array}$ & $\begin{array}{l}\text { Mao Ci } \\
\text { Jin Ji Er }\end{array}$ & $\begin{array}{l}\text { Tie mao tou, Hei mao tou, } \\
\text { Da mao tou }\end{array}$ & Shrub & $\begin{array}{l}\text { Stem, } \\
\text { leaf, } \\
\text { flower }\end{array}$ & 0 & 0 & 60 & 39 & 0 & 27 & 126 & 40 \\
\hline Leguminosae & $\begin{array}{l}\text { Glycyrrhiza } \\
\text { uralensis Fisch. }\end{array}$ & $\begin{array}{l}\text { Gan } \\
\text { Cao }\end{array}$ & Gan cao & Herb & $\begin{array}{l}\text { Stem, } \\
\text { leaf, } \\
\text { fruit }\end{array}$ & 26 & 6 & 2 & 8 & 0 & 37 & 79 & 25.08 \\
\hline Leguminosae & $\begin{array}{l}\text { Gueldenstaedtia } \\
\text { stenophylla Bunge }\end{array}$ & $\begin{array}{l}\text { Xia Ye } \\
\text { Mi Kou } \\
\text { Dai }\end{array}$ & $\begin{array}{l}\text { Mi gu zhuang zhuang, } \\
\text { Liang shi zhuang zhuang }\end{array}$ & Herb & $\begin{array}{l}\text { Leaf, } \\
\text { flower, } \\
\text { fruit }\end{array}$ & 0 & 0 & 43 & 19 & 1 & 0 & 63 & 20 \\
\hline Leguminosae & $\begin{array}{l}\text { Gueldenstaedtia } \\
\text { verna (Georgi) } \\
\text { Boriss. }\end{array}$ & $\begin{array}{l}\text { Shao } \\
\text { Hua Mi } \\
\text { Kou Dai }\end{array}$ & Mi gu zhuang zhuang & Herb & $\begin{array}{l}\text { Stem, } \\
\text { leaf }\end{array}$ & 0 & 0 & 0 & 0 & 2 & 0 & 2 & 0.63 \\
\hline Leguminosae & $\begin{array}{l}\text { Lathyrus davidii } \\
\text { Hance }\end{array}$ & $\begin{array}{l}\text { Da Shan } \\
\text { Li Dou }\end{array}$ & Jing er wan & Herb & $\begin{array}{l}\text { Stem, } \\
\text { leaf }\end{array}$ & 0 & 0 & 0 & 0 & 21 & 0 & 21 & 6.67 \\
\hline Leguminosae & $\begin{array}{l}\text { Lathyrus } \\
\text { quinquenervius } \\
\text { (Miq.) Litv. }\end{array}$ & $\begin{array}{l}\text { Shan Li } \\
\text { Dou }\end{array}$ & Jing er wan & Herb & $\begin{array}{l}\text { Stem, } \\
\text { leaf }\end{array}$ & 0 & 0 & 0 & 0 & 20 & 0 & 20 & 6.35 \\
\hline Leguminosae & $\begin{array}{l}\text { Lens culinaris } \\
\text { Medik. }\end{array}$ & Bin Dou & Bian dou zi, Xiao bian dou & Herb & $\begin{array}{l}\text { Stem, } \\
\text { leaf }\end{array}$ & 0 & 0 & 0 & 0 & 0 & 1 & 1 & 0.32 \\
\hline Leguminosae & $\begin{array}{l}\text { Lespedeza davurica } \\
\text { (Laxm.) Schindl. }\end{array}$ & $\begin{array}{l}\text { Xin An } \\
\mathrm{Hu} Z \mathrm{Zhi} \\
\mathrm{Zi}\end{array}$ & Hu shi tiao & Shrub & $\begin{array}{l}\text { Flower, } \\
\text { fruit }\end{array}$ & 0 & 6 & 55 & 47 & 0 & 34 & 142 & 45.08 \\
\hline Leguminosae & $\begin{array}{l}\text { Lespedeza potaninii } \\
\text { Vassilcz. }\end{array}$ & $\begin{array}{l}\mathrm{Niu} Z \mathrm{Zhi} \\
\mathrm{Zi}\end{array}$ & Hu shi tiao & Shrub & $\begin{array}{l}\text { Flower, } \\
\text { fruit }\end{array}$ & 0 & 6 & 56 & 46 & 0 & 34 & 142 & 45.08 \\
\hline Leguminosae & Medicago falcata L. & $\begin{array}{l}\text { Ye Mu } \\
\mathrm{Xu}\end{array}$ & Huang hua ye mu xu & Herb & $\begin{array}{l}\text { Stem, } \\
\text { leaf }\end{array}$ & 6 & 0 & 45 & 47 & 38 & 40 & 176 & 55.87 \\
\hline Leguminosae & $\begin{array}{l}\text { Medicago lupulina } \\
\text { L. }\end{array}$ & $\begin{array}{l}\text { Tian Lan } \\
\text { Mu Xu }\end{array}$ & Di mu xu & Herb & $\begin{array}{l}\text { Stem, } \\
\text { leaf }\end{array}$ & 0 & 0 & 0 & 0 & 38 & 0 & 38 & 12.06 \\
\hline Leguminosae & $\begin{array}{l}\text { Medicago ruthenica } \\
\text { (L.) Trautv. }\end{array}$ & $\begin{array}{l}\text { Hua Mu } \\
\text { Xu }\end{array}$ & $\begin{array}{l}\text { Ye mu xu, Qiao pi cao, Di } \\
\text { mu xu }\end{array}$ & Herb & $\begin{array}{l}\text { Stem, } \\
\text { leaf }\end{array}$ & 0 & 0 & 0 & 0 & 38 & 40 & 78 & 24.76 \\
\hline Leguminosae & $\begin{array}{l}\text { Medicago ruthenica } \\
\text { (L.)Ledeb. }\end{array}$ & $\begin{array}{l}\text { Zi Hua } \\
\text { Mu Xu }\end{array}$ & Zi hua mu xu & Herb & $\begin{array}{l}\text { Stem, } \\
\text { leaf }\end{array}$ & 29 & 16 & 44 & 49 & 36 & 40 & 214 & 67.94 \\
\hline Leguminosae & $\begin{array}{l}\text { Melilotus albus } \\
\text { Medik. }\end{array}$ & $\begin{array}{l}\text { Bai Hua } \\
\text { Cao Mu } \\
\text { Xi }\end{array}$ & Ma mu xu & Herb & $\begin{array}{l}\text { Stem, } \\
\text { leaf }\end{array}$ & 0 & 0 & 0 & 0 & 0 & 39 & 39 & 12.38 \\
\hline Leguminosae & $\begin{array}{l}\text { Melilotus officinalis } \\
\text { (L.) Pall. }\end{array}$ & $\begin{array}{l}\text { Huang } \\
\text { Hua } \\
\text { Cao Mu } \\
\text { Xi }\end{array}$ & Ma mu xu & Herb & $\begin{array}{l}\text { Stem, } \\
\text { leaf }\end{array}$ & 0 & 0 & 0 & 0 & 0 & 39 & 39 & 12.38 \\
\hline Leguminosae & $\begin{array}{l}\text { Onobrychis viciifolia } \\
\text { Scop. }\end{array}$ & $\begin{array}{l}\text { Lv Shi } \\
\text { Cao }\end{array}$ & Hong dou cao & Herb & $\begin{array}{l}\text { Stem, } \\
\text { leaf }\end{array}$ & 2 & 0 & 0 & 0 & 36 & 0 & 38 & 12.06 \\
\hline Leguminosae & $\begin{array}{l}\text { Oxytropis aciphylla } \\
\text { Ledeb. }\end{array}$ & $\begin{array}{l}\text { Ci Ye } \\
\text { Bing Ji } \\
\text { Dou }\end{array}$ & $\begin{array}{l}\text { Mao tou chai, Xiao mao } \\
\text { tou }\end{array}$ & Shrub & $\begin{array}{l}\text { Stem, } \\
\text { leaf, } \\
\text { flower }\end{array}$ & 0 & 0 & 60 & 44 & 0 & 27 & 131 & 41.59 \\
\hline Leguminosae & Pisum sativum L. & $\begin{array}{l}\text { Wan } \\
\text { Dou }\end{array}$ & Wan dou & Herb & $\begin{array}{l}\text { Stem, } \\
\text { leaf, } \\
\text { fruit }\end{array}$ & 0 & 0 & 0 & 0 & 4 & 0 & 4 & 1.27 \\
\hline Leguminosae & $\begin{array}{l}\text { Robinia } \\
\text { pseudoacacia L. }\end{array}$ & Ci Huai & Yang huai, Huai shu & Tree & $\begin{array}{l}\text { Stem, } \\
\text { leaf }\end{array}$ & 14 & 0 & 15 & 0 & 1 & 0 & 30 & 9.52 \\
\hline Leguminosae & Sophora & Ku Dou & Ku du zi, Ye hu wan dou & Shrub & Fruit & 0 & 0 & 3 & 0 & 0 & 30 & 33 & 10.48 \\
\hline
\end{tabular}


Table 4 Traditional forage plants and their relative frequency of citation (RFC) (Continued)

\begin{tabular}{|c|c|c|c|c|c|c|c|c|c|c|c|c|c|}
\hline \multirow[t]{2}{*}{ Family name } & \multirow[t]{2}{*}{ Scientific name } & \multirow[t]{2}{*}{$\begin{array}{l}\text { Chinese } \\
\text { name }\end{array}$} & \multirow[t]{2}{*}{ Local name } & \multirow[t]{2}{*}{$\begin{array}{l}\text { Life } \\
\text { form }\end{array}$} & \multirow[t]{2}{*}{$\begin{array}{l}\text { Part } \\
\text { used }\end{array}$} & \multicolumn{6}{|c|}{$\begin{array}{l}\text { Frequency of citations } \\
\text { in the surveyed villages }\end{array}$} & \multirow[t]{2}{*}{$\begin{array}{l}\text { Frequency } \\
\text { of citation (FC) }\end{array}$} & \multirow{2}{*}{$\begin{array}{l}\text { Relative } \\
\text { frequency } \\
\text { of citation } \\
\text { (RFC) }\end{array}$} \\
\hline & & & & & & a & $b$ & c & $d$ & e & f & & \\
\hline & alopecuroides L. & $\mathrm{Zi}$ & & & & & & & & & & & \\
\hline Leguminosae & $\begin{array}{l}\text { Styphnolobium } \\
\text { japonicum (L.) } \\
\text { Schott }\end{array}$ & Huai & Huai shu & Tree & $\begin{array}{l}\text { Stem, } \\
\text { leaf }\end{array}$ & 0 & 0 & 15 & 0 & 1 & 0 & 16 & 5.08 \\
\hline Leguminosae & Trifolium repens $\mathrm{L}$. & $\begin{array}{l}\text { Bai Che } \\
\text { Zhou } \\
\text { Cao }\end{array}$ & Bai hua ye mu xu & Herb & $\begin{array}{l}\text { Stem, } \\
\text { leaf }\end{array}$ & 0 & 0 & 34 & 8 & 38 & 0 & 80 & 25.4 \\
\hline Leguminosae & $\begin{array}{l}\text { Vicia amoena } \\
\text { Fisch. }\end{array}$ & $\begin{array}{l}\text { Shan Ye } \\
\text { Wan } \\
\text { Dou }\end{array}$ & Ye wan dou & Herb & $\begin{array}{l}\text { Stem, } \\
\text { leaf }\end{array}$ & 0 & 0 & 0 & 0 & 29 & 0 & 29 & 9.21 \\
\hline Leguminosae & Vicia cracca L. & $\begin{array}{l}\text { Guang } \\
\text { Bu Ye } \\
\text { Wan } \\
\text { Dou }\end{array}$ & $\begin{array}{l}\text { Luo dou yang, Ye wan } \\
\text { dou }\end{array}$ & Herb & $\begin{array}{l}\text { Stem, } \\
\text { leaf }\end{array}$ & 0 & 0 & 3 & 1 & 29 & 1 & 34 & 10.79 \\
\hline Leguminosae & Vicia faba $\mathrm{L}$. & $\begin{array}{l}\text { Can } \\
\text { Dou }\end{array}$ & Da dou & Herb & $\begin{array}{l}\text { Stem, } \\
\text { leaf }\end{array}$ & 8 & 0 & 3 & 7 & 13 & 1 & 32 & 10.16 \\
\hline Leguminosae & Vicia sepium L. & $\begin{array}{l}\text { Ye Wan } \\
\text { Dou }\end{array}$ & Ye wan dou & Herb & $\begin{array}{l}\text { Stem, } \\
\text { leaf }\end{array}$ & 0 & 0 & 0 & 0 & 29 & 0 & 29 & 9.21 \\
\hline Leguminosae & Vicia unijuga A.Br. & $\begin{array}{l}\text { Wai Tou } \\
\text { Cai }\end{array}$ & Wai tou cai & Herb & $\begin{array}{l}\text { Stem, } \\
\text { leaf }\end{array}$ & 0 & 0 & 0 & 0 & 10 & 0 & 10 & 3.17 \\
\hline Rosaceae & $\begin{array}{l}\text { Potentilla anserina } \\
\text { L. }\end{array}$ & $\begin{array}{l}\text { E Rong } \\
\text { Wei } \\
\text { Ling Cai }\end{array}$ & Jue ma & Herb & Root & 0 & 0 & 0 & 0 & 4 & 0 & 4 & 1.27 \\
\hline Rosaceae & $\begin{array}{l}\text { Sibbaldianthe } \\
\text { bifurca (L.) Kurtto \& } \\
\text { T.Erikss. }\end{array}$ & $\begin{array}{l}\text { Er Lie } \\
\text { Wei } \\
\text { Ling Cai }\end{array}$ & $\begin{array}{l}\text { Tie pian zi, Hei gen zi } \\
\text { yang, Ji guan cao }\end{array}$ & $\begin{array}{l}\text { Herb } \\
\text { or } \\
\text { Shrub }\end{array}$ & $\begin{array}{l}\text { Stem, } \\
\text { leaf }\end{array}$ & 0 & 0 & 53 & 26 & 5 & 35 & 119 & 37.78 \\
\hline Rosaceae & $\begin{array}{l}\text { Crataegus } \\
\text { pinnatifida Bunge }\end{array}$ & $\begin{array}{l}\text { Shan } \\
\text { Zha }\end{array}$ & Shan cha shu & Tree & Leaf & 0 & 0 & 0 & 0 & 2 & 0 & 2 & 0.63 \\
\hline Rosaceae & $\begin{array}{l}\text { Duchesnea indica } \\
\text { (Jacks.) Focke }\end{array}$ & She Mei & Pie er, Mei zi & Herb & $\begin{array}{l}\text { Stem, } \\
\text { leaf }\end{array}$ & 0 & 0 & 0 & 0 & 2 & 0 & 2 & 0.63 \\
\hline Rosaceae & $\begin{array}{l}\text { Fragaria orientalis } \\
\text { Losinsk. }\end{array}$ & $\begin{array}{l}\text { Dong } \\
\text { Fang } \\
\text { Cao Mei }\end{array}$ & Ye cao mei, Pie er & Herb & $\begin{array}{l}\text { Stem, } \\
\text { leaf }\end{array}$ & 0 & 0 & 0 & 0 & 2 & 0 & 2 & 0.63 \\
\hline Rosaceae & Potentilla acaulis L. & $\begin{array}{l}\text { Xing } \\
\text { Mao } \\
\text { Wei } \\
\text { Ling Cai }\end{array}$ & Ma shi shi & Herb & Leaf & 0 & 0 & 0 & 0 & 0 & 12 & 12 & 3.81 \\
\hline Rosaceae & Potentilla reptans $\mathrm{L}$. & $\begin{array}{l}\text { Pu Fu } \\
\text { Wei } \\
\text { Ling Cai }\end{array}$ & Hong bang chui & Herb & Leaf & 0 & 0 & 0 & 0 & 14 & 0 & 14 & 4.44 \\
\hline Rosaceae & $\begin{array}{l}\text { Prinsepia uniflora } \\
\text { Batalin }\end{array}$ & Rui He & Ma ru ci, Ma ru zi & Shrub & Leaf & 0 & 0 & 53 & 36 & 0 & 0 & 89 & 28.25 \\
\hline Rosaceae & $\begin{array}{l}\text { Prunus davidiana } \\
\text { (Carrišre) Franch. }\end{array}$ & $\begin{array}{l}\text { Shan } \\
\text { Tao }\end{array}$ & Shan mao tao, Ye tao & $\begin{array}{l}\text { Shrub } \\
\text { or } \\
\text { Tree }\end{array}$ & $\begin{array}{l}\text { Stem, } \\
\text { leaf }\end{array}$ & 0 & 0 & 3 & 26 & 1 & 21 & 51 & 16.19 \\
\hline Rosaceae & Prunus sibirica L. & $\begin{array}{l}\text { Shan } \\
\text { Xing }\end{array}$ & Xing zi, Heng zi & $\begin{array}{l}\text { Shrub } \\
\text { or } \\
\text { Tree }\end{array}$ & $\begin{array}{l}\text { Stem, } \\
\text { leaf }\end{array}$ & 0 & 0 & 11 & 26 & 1 & 23 & 61 & 19.37 \\
\hline Rosaceae & $\begin{array}{l}\text { Prunus tomentosa } \\
\text { Thunb. }\end{array}$ & $\begin{array}{l}\text { Mao } \\
\text { Ying } \\
\text { Tao }\end{array}$ & Shan yin tao, Yin tao & Shrub & Leaf & 0 & 0 & 0 & 0 & 1 & 0 & 1 & 0.32 \\
\hline Rosaceae & Rubus parvifolius L. & $\begin{array}{l}\text { Mao } \\
\text { Mei }\end{array}$ & Mei dou wan & Shrub & Leaf & 0 & 0 & 0 & 0 & 8 & 0 & 8 & 2.54 \\
\hline Rosaceae & Rubus parvifolius & Xian & Mei dou wan & Herb & Leaf & 0 & 0 & 0 & 0 & 8 & 0 & 8 & 2.54 \\
\hline
\end{tabular}


Table 4 Traditional forage plants and their relative frequency of citation (RFC) (Continued)

\begin{tabular}{|c|c|c|c|c|c|c|c|c|c|c|c|c|c|}
\hline \multirow[t]{2}{*}{ Family name } & \multirow[t]{2}{*}{ Scientific name } & \multirow[t]{2}{*}{$\begin{array}{l}\text { Chinese } \\
\text { name }\end{array}$} & \multirow[t]{2}{*}{ Local name } & \multirow[t]{2}{*}{$\begin{array}{l}\text { Life } \\
\text { form }\end{array}$} & \multirow[t]{2}{*}{$\begin{array}{l}\text { Part } \\
\text { used }\end{array}$} & \multicolumn{6}{|c|}{$\begin{array}{l}\text { Frequency of citations } \\
\text { in the surveyed villages }\end{array}$} & \multirow[t]{2}{*}{$\begin{array}{l}\text { Frequency } \\
\text { of citation (FC) }\end{array}$} & \multirow{2}{*}{$\begin{array}{l}\text { Relative } \\
\text { frequency } \\
\text { of citation } \\
\text { (RFC) }\end{array}$} \\
\hline & & & & & & a & $b$ & c & $d$ & e & $f$ & & \\
\hline & $\begin{array}{l}\text { var. taquetii (H. } \\
\text { Lév.) Lauener \& } \\
\text { D.K. Ferguson }\end{array}$ & $\begin{array}{l}\text { Hua } \\
\text { Mao } \\
\text { Mei }\end{array}$ & & $\begin{array}{l}\text { or } \\
\text { Shrub }\end{array}$ & & & & & & & & & \\
\hline Rosaceae & $\begin{array}{l}\text { Rubus pungens } \\
\text { Cambess. }\end{array}$ & $\begin{array}{l}\text { Zhen Ci } \\
\text { Xuan } \\
\text { Gou Zi }\end{array}$ & Hei mei dou & Shrub & $\begin{array}{l}\text { Stem, } \\
\text { leaf }\end{array}$ & 0 & 0 & 0 & 0 & 4 & 0 & 4 & 1.27 \\
\hline Rosaceae & $\begin{array}{l}\text { Spiraea pubescens } \\
\text { Turcz. }\end{array}$ & $\begin{array}{l}\text { Tu } \\
\text { Zhuang } \\
\text { Xiu Xian } \\
\text { Ju }\end{array}$ & Gan you ben zi & Shrub & Leaf & 0 & 0 & 0 & 0 & 8 & 0 & 8 & 2.54 \\
\hline Elaeagnaceae & $\begin{array}{l}\text { Elaeagnus } \\
\text { angustifolia L. }\end{array}$ & Sha Zao & Sha zao zi & $\begin{array}{l}\text { Shrub } \\
\text { or } \\
\text { Tree }\end{array}$ & $\begin{array}{l}\text { Stem, } \\
\text { leaf }\end{array}$ & 0 & 0 & 3 & 27 & 0 & 0 & 30 & 9.52 \\
\hline Elaeagnaceae & $\begin{array}{l}\text { Elaeagnus } \\
\text { rhamnoides (L.) } \\
\text { A.Nelson }\end{array}$ & Sha Ji & Sha bing, Hei ci & Shrub & $\begin{array}{l}\text { Stem, } \\
\text { leaf }\end{array}$ & 0 & 0 & 32 & 31 & 12 & 1 & 76 & 24.13 \\
\hline Rhamnaceae & $\begin{array}{l}\text { Ziziphus jujuba var. } \\
\text { spinosa (Bunge) } \\
\text { Hu ex H.F.Chow }\end{array}$ & $\begin{array}{l}\text { Suan } \\
\text { Zao }\end{array}$ & Suan zao zi, Shan zao zi & $\begin{array}{l}\text { Shrub } \\
\text { or } \\
\text { Tree }\end{array}$ & $\begin{array}{l}\text { Stem, } \\
\text { leaf }\end{array}$ & 0 & 0 & 3 & 33 & 0 & 0 & 36 & 11.43 \\
\hline Ulmaceae & Ulmus pumila L. & Yu Shu & Yu shu & Tree & Leaf & 8 & 0 & 29 & 20 & 1 & 0 & 58 & 18.41 \\
\hline Moraceae & Morus alba L. & Sang & Sang shu & $\begin{array}{l}\text { Shrub } \\
\text { or } \\
\text { Tree }\end{array}$ & $\begin{array}{l}\text { Stem, } \\
\text { leaf }\end{array}$ & 0 & 0 & 0 & 0 & 1 & 0 & 1 & 0.32 \\
\hline Cucurbitaceae & $\begin{array}{l}\text { Citrullus lanatus } \\
\text { (Thunb.) Matsum. } \\
\text { \& Nakai }\end{array}$ & Xi Gua & Xi gua yang & Liana & $\begin{array}{l}\text { Stem, } \\
\text { leaf }\end{array}$ & 0 & 0 & 1 & 44 & 0 & 0 & 45 & 14.29 \\
\hline Violaceae & $\begin{array}{l}\text { Viola philippica } \\
\text { Cav. }\end{array}$ & $\begin{array}{l}\text { Zi Hua } \\
\text { Di Ding }\end{array}$ & Dao jian yao & Herb & $\begin{array}{l}\text { Stem, } \\
\text { leaf }\end{array}$ & 0 & 0 & 0 & 0 & 6 & 0 & 6 & 1.9 \\
\hline Linaceae & $\begin{array}{l}\text { Linum pallescens } \\
\text { Bunge }\end{array}$ & $\begin{array}{l}\text { Duan } \\
\text { Zhu Ya } \\
\text { Ma }\end{array}$ & Ye hu ma & Herb & $\begin{array}{l}\text { Flower, } \\
\text { fruit }\end{array}$ & 0 & 0 & 50 & 39 & 0 & 0 & 89 & 28.25 \\
\hline Linaceae & Linum perenne L. & $\begin{array}{l}\text { Su Gen } \\
\text { Ya Ma }\end{array}$ & Ye hu ma & Herb & $\begin{array}{l}\text { Flower, } \\
\text { fruit }\end{array}$ & 0 & 0 & 0 & 0 & 0 & 11 & 11 & 3.49 \\
\hline Linaceae & $\begin{array}{l}\text { Linum stelleroides } \\
\text { Planch. }\end{array}$ & $\begin{array}{l}\text { Ye Ya } \\
\mathrm{Ma}\end{array}$ & Ye hu ma & Herb & $\begin{array}{l}\text { Stem, } \\
\text { leaf, } \\
\text { flower, } \\
\text { fruit }\end{array}$ & 0 & 0 & 50 & 39 & 1 & 0 & 90 & 28.57 \\
\hline Linaceae & $\begin{array}{l}\text { Linum } \\
\text { usitatissimum L. }\end{array}$ & Ya Ma & Jing zi hu ma & Herb & $\begin{array}{l}\text { Stem, } \\
\text { leaf, } \\
\text { flower, } \\
\text { fruit }\end{array}$ & 0 & 0 & 16 & 10 & 1 & 13 & 40 & 12.7 \\
\hline Geraniaceae & $\begin{array}{l}\text { Erodium } \\
\text { stephanianum } \\
\text { Willd. }\end{array}$ & $\begin{array}{l}\text { Mang } \\
\text { Niu Er } \\
\text { Miao }\end{array}$ & Hong gen zi & Herb & $\begin{array}{l}\text { Stem, } \\
\text { leaf, } \\
\text { flower }\end{array}$ & 0 & 0 & 39 & 10 & 0 & 33 & 82 & 26.03 \\
\hline Geraniaceae & $\begin{array}{l}\text { Geranium sibiricum } \\
\mathrm{L} \text {. }\end{array}$ & $\begin{array}{l}\text { Shu } \\
\text { Zhang } \\
\text { Lao } \\
\text { Guan } \\
\text { Cao }\end{array}$ & Lao guan cao & Herb & $\begin{array}{l}\text { Stem, } \\
\text { leaf, } \\
\text { flower }\end{array}$ & 0 & 0 & 0 & 0 & 6 & 0 & 6 & 1.9 \\
\hline Nitrariaceae & $\begin{array}{l}\text { Nitraria } \\
\text { tangutorum } \\
\text { Bobrov }\end{array}$ & Bai Ci & $\begin{array}{l}\text { bia ci, Suan liu zi, Gai lia zi, } \\
\text { Ga la mu }\end{array}$ & Shrub & $\begin{array}{l}\text { Stem, } \\
\text { leaf }\end{array}$ & 0 & 0 & 2 & 19 & 0 & 24 & 45 & 14.29 \\
\hline Nitrariaceae & $\begin{array}{l}\text { Peganum harmala } \\
\mathrm{L} \text {. }\end{array}$ & $\begin{array}{l}\text { Luo Tuo } \\
\text { Peng }\end{array}$ & $\begin{array}{l}\text { Luo tuo peng, Luo luo } \\
\text { peng }\end{array}$ & Herb & $\begin{array}{l}\text { Stem, } \\
\text { leaf }\end{array}$ & 0 & 10 & 59 & 45 & 0 & 35 & 149 & 47.3 \\
\hline Nitrariaceae & Peganum & Duo Lie & Luo tuo peng, Luo luo & Herb & Stem, & 0 & 10 & 59 & 45 & 0 & 35 & 149 & 47.3 \\
\hline
\end{tabular}


Table 4 Traditional forage plants and their relative frequency of citation (RFC) (Continued)

\begin{tabular}{|c|c|c|c|c|c|c|c|c|c|c|c|c|c|}
\hline \multirow[t]{2}{*}{ Family name } & \multirow[t]{2}{*}{ Scientific name } & \multirow[t]{2}{*}{$\begin{array}{l}\text { Chinese } \\
\text { name }\end{array}$} & \multirow[t]{2}{*}{ Local name } & \multirow[t]{2}{*}{$\begin{array}{l}\text { Life } \\
\text { form }\end{array}$} & \multirow[t]{2}{*}{$\begin{array}{l}\text { Part } \\
\text { used }\end{array}$} & \multicolumn{6}{|c|}{$\begin{array}{l}\text { Frequency of citations } \\
\text { in the surveyed villages }\end{array}$} & \multirow[t]{2}{*}{$\begin{array}{l}\text { Frequency } \\
\text { of citation (FC) }\end{array}$} & \multirow{2}{*}{$\begin{array}{l}\text { Relative } \\
\text { frequency } \\
\text { of citation } \\
\text { (RFC) }\end{array}$} \\
\hline & & & & & & a & $b$ & c & $d$ & e & $f$ & & \\
\hline & $\begin{array}{l}\text { multisectum } \\
\text { (Maxim.) Bobrov }\end{array}$ & $\begin{array}{l}\text { Luo Tuo } \\
\text { Peng }\end{array}$ & peng & & leaf & & & & & & & & \\
\hline Nitrariaceae & $\begin{array}{l}\text { Peganum } \\
\text { nigellastrum Bunge }\end{array}$ & $\begin{array}{l}\text { Luo Tuo } \\
\text { Hao }\end{array}$ & Xiao luo tuo peng & Herb & $\begin{array}{l}\text { Stem, } \\
\text { leaf }\end{array}$ & 0 & 0 & 59 & 45 & 0 & 0 & 104 & 33.02 \\
\hline Rutaceae & $\begin{array}{l}\text { Haplophyllum } \\
\text { dauricum (L.) G. } \\
\text { Don }\end{array}$ & $\begin{array}{l}\text { Bei Yun } \\
\text { Xiang }\end{array}$ & Huang hua hua & Herb & $\begin{array}{l}\text { Stem, } \\
\text { leaf, } \\
\text { flower }\end{array}$ & 0 & 0 & 50 & 39 & 0 & 0 & 89 & 28.25 \\
\hline Malvaceae & Alcea rosea $\mathrm{L}$. & Shu Kui & Ye wei hua & Herb & $\begin{array}{l}\text { Stem, } \\
\text { leaf, } \\
\text { flower }\end{array}$ & 0 & 0 & 0 & 0 & 12 & 0 & 12 & 3.81 \\
\hline Malvaceae & Hibiscus trionum L. & $\begin{array}{l}\text { Ye Xi } \\
\text { Gua } \\
\text { Miao }\end{array}$ & Hei zi zi & Herb & $\begin{array}{l}\text { Stem, } \\
\text { leaf }\end{array}$ & 0 & 0 & 0 & 0 & 0 & 6 & 6 & 1.9 \\
\hline Malvaceae & Malva verticillata $L$. & $\begin{array}{l}\text { Dong } \\
\text { Kui }\end{array}$ & Qi ye zi, Ye jing kui & Herb & $\begin{array}{l}\text { Stem, } \\
\text { leaf }\end{array}$ & 3 & 0 & 0 & 0 & 2 & 26 & 31 & 9.84 \\
\hline Brassicaceae & $\begin{array}{l}\text { Braya humilis (C.A. } \\
\text { Mey.) B.L. Rob. }\end{array}$ & $\begin{array}{l}\text { Yin Guo } \\
\text { Jie }\end{array}$ & Bai hua zi, Que er nao nao & Herb & $\begin{array}{l}\text { Stem, } \\
\text { leaf }\end{array}$ & 0 & 0 & 47 & 33 & 0 & 33 & 113 & 35.87 \\
\hline Brassicaceae & $\begin{array}{l}\text { Capsella bursa- } \\
\text { pastoris (L.) Medik. }\end{array}$ & $\mathrm{Ji}$ & Hua hua cai, Ji cai & Herb & $\begin{array}{l}\text { Stem, } \\
\text { leaf }\end{array}$ & 0 & 0 & 0 & 0 & 1 & 0 & 1 & 0.32 \\
\hline Brassicaceae & $\begin{array}{l}\text { Descurainia sophia } \\
\text { (L.) Webb ex Prantl }\end{array}$ & $\begin{array}{l}\text { Bo } \\
\text { Niang } \\
\text { HaO }\end{array}$ & Ye cai zi & Herb & $\begin{array}{l}\text { Stem, } \\
\text { leaf }\end{array}$ & 0 & 0 & 0 & 0 & 18 & 0 & 18 & 5.71 \\
\hline Brassicaceae & Draba nemorosa L. & Ting Li & Niu ji jiao & Herb & $\begin{array}{l}\text { Stem, } \\
\text { leaf }\end{array}$ & 0 & 0 & 0 & 0 & 21 & 0 & 21 & 6.67 \\
\hline Brassicaceae & $\begin{array}{l}\text { Eruca vesicaria (L.) } \\
\text { Cav. }\end{array}$ & $\begin{array}{l}\text { Zhi Ma } \\
\text { Cai }\end{array}$ & Yun gai, Yuan yuan & Herb & Seed & 0 & 0 & 21 & 8 & 9 & 27 & 65 & 20.63 \\
\hline Brassicaceae & $\begin{array}{l}\text { Lepidium apetalum } \\
\text { Willd. }\end{array}$ & $\begin{array}{l}\text { Du Xing } \\
\text { Cai }\end{array}$ & La la yang, La la ying & Herb & $\begin{array}{l}\text { Stem, } \\
\text { leaf }\end{array}$ & 0 & 11 & 47 & 26 & 7 & 22 & 113 & 35.87 \\
\hline Brassicaceae & $\begin{array}{l}\text { Lepidium latifolium } \\
\text { L. }\end{array}$ & $\begin{array}{l}\text { Kuan Ye } \\
\text { Du Xing } \\
\text { Cai }\end{array}$ & Da la la & Herb & $\begin{array}{l}\text { Stem, } \\
\text { leaf }\end{array}$ & 0 & 0 & 0 & 0 & 0 & 14 & 14 & 4.44 \\
\hline Brassicaceae & $\begin{array}{l}\text { Malcolmia africana } \\
\text { (L.) R.Br. }\end{array}$ & Se Ji & Tian luo bo & Herb & $\begin{array}{l}\text { Stem, } \\
\text { leaf }\end{array}$ & 0 & 0 & 0 & 0 & 0 & 20 & 20 & 6.35 \\
\hline Brassicaceae & Thlaspi arvense L. & Xi Mi & Ku gai zi & Herb & $\begin{array}{l}\text { Stem, } \\
\text { leaf }\end{array}$ & 0 & 0 & 0 & 0 & 1 & 0 & 1 & 0.32 \\
\hline Tamaricaceae & $\begin{array}{l}\text { Reaumuria } \\
\text { soongarica }\end{array}$ & $\begin{array}{l}\text { Hong } \\
\text { Sha }\end{array}$ & $\begin{array}{l}\text { Hong xiang chai, Hong } \\
\text { xun chai }\end{array}$ & Shrub & $\begin{array}{l}\text { Stem, } \\
\text { leaf }\end{array}$ & 0 & 0 & 48 & 38 & 0 & 0 & 86 & 27.3 \\
\hline Tamaricaceae & $\begin{array}{l}\text { Tamarix chinensis } \\
\text { Lour. }\end{array}$ & Guai Liu & Guai liu, Hong liu & $\begin{array}{l}\text { Shrub } \\
\text { or } \\
\text { Tree }\end{array}$ & $\begin{array}{l}\text { Stem, } \\
\text { leaf, } \\
\text { flower }\end{array}$ & 0 & 0 & 4 & 9 & 0 & 0 & 13 & 4.13 \\
\hline Plumbaginaceae & $\begin{array}{l}\text { Limonium bicolor } \\
\text { (Bunge) Kuntze }\end{array}$ & $\begin{array}{l}\text { Er Se Bu } \\
\text { Xue Cao }\end{array}$ & Xiao hua hua, Ma niu niu & Herb & $\begin{array}{l}\text { Leaf, } \\
\text { flower }\end{array}$ & 0 & 0 & 29 & 20 & 0 & 22 & 71 & 22.54 \\
\hline Polygonaceae & $\begin{array}{l}\text { Calligonum } \\
\text { mongolicum Turcz. }\end{array}$ & $\begin{array}{l}\text { Sha } \\
\text { Guai } \\
\text { Zao }\end{array}$ & Sha zao zi & Shrub & $\begin{array}{l}\text { Stem, } \\
\text { leaf, } \\
\text { fruit }\end{array}$ & 0 & 0 & 9 & 43 & 0 & 0 & 52 & 16.51 \\
\hline Polygonaceae & $\begin{array}{l}\text { Fagopyrum } \\
\text { esculentum } \\
\text { Moench }\end{array}$ & $\begin{array}{l}\text { Qiao } \\
\text { Mai }\end{array}$ & Qiao mai, Tian qiao & Herb & $\begin{array}{l}\text { Stem, } \\
\text { leaf }\end{array}$ & 6 & 0 & 4 & 3 & 1 & 1 & 15 & 4.76 \\
\hline Polygonaceae & $\begin{array}{l}\text { Fagopyrum } \\
\text { tataricum (L.) } \\
\text { Gaertn. }\end{array}$ & Ku Qiao & Ku qiao & Herb & $\begin{array}{l}\text { Stem, } \\
\text { leaf }\end{array}$ & 0 & 0 & 14 & 3 & 1 & 1 & 19 & 6.03 \\
\hline Polygonaceae & $\begin{array}{l}\text { Persicaria } \\
\text { lapathifolia (L.) } \\
\text { Delarbre }\end{array}$ & $\begin{array}{l}\text { Suan } \\
\text { Mo Ye } \\
\text { Liao }\end{array}$ & $\begin{array}{l}\text { Da ye suan bu liu liu, Da } \\
\text { ma liao }\end{array}$ & Herb & $\begin{array}{l}\text { Stem, } \\
\text { leaf }\end{array}$ & 0 & 0 & 0 & 0 & 15 & 0 & 15 & 4.76 \\
\hline
\end{tabular}


Table 4 Traditional forage plants and their relative frequency of citation (RFC) (Continued)

\begin{tabular}{|c|c|c|c|c|c|c|c|c|c|c|c|c|c|}
\hline \multirow[t]{2}{*}{ Family name } & \multirow[t]{2}{*}{ Scientific name } & \multirow[t]{2}{*}{$\begin{array}{l}\text { Chinese } \\
\text { name }\end{array}$} & \multirow[t]{2}{*}{ Local name } & \multirow[t]{2}{*}{$\begin{array}{l}\text { Life } \\
\text { form }\end{array}$} & \multirow[t]{2}{*}{$\begin{array}{l}\text { Part } \\
\text { used }\end{array}$} & \multicolumn{6}{|c|}{$\begin{array}{l}\text { Frequency of citations } \\
\text { in the surveyed villages }\end{array}$} & \multirow[t]{2}{*}{$\begin{array}{l}\text { Frequency } \\
\text { of citation (FC) }\end{array}$} & \multirow{2}{*}{$\begin{array}{l}\text { Relative } \\
\text { frequency } \\
\text { of citation } \\
\text { (RFC) }\end{array}$} \\
\hline & & & & & & $\mathrm{a}$ & $\mathrm{b}$ & c & $d$ & e & $f$ & & \\
\hline Polygonaceae & $\begin{array}{l}\text { Persicaria vivipara } \\
\text { (L.) Ronse Decr. }\end{array}$ & $\begin{array}{l}\text { Zhu Ya } \\
\text { Liao }\end{array}$ & $\begin{array}{l}\text { Tie xiu xiu, Hong san qi, } \\
\text { Qiao mai qi }\end{array}$ & Herb & $\begin{array}{l}\text { Stem, } \\
\text { leaf }\end{array}$ & 0 & 0 & 51 & 43 & 1 & 0 & 95 & 30.16 \\
\hline Polygonaceae & $\begin{array}{l}\text { Polygonum } \\
\text { aviculare L. }\end{array}$ & Bian Xu & Bian xu zi, Ye sao zhou & Herb & $\begin{array}{l}\text { Stem, } \\
\text { leaf }\end{array}$ & 0 & 0 & 51 & 43 & 2 & 10 & 106 & 33.65 \\
\hline Polygonaceae & $\begin{array}{l}\text { Polygonum } \\
\text { sibiricum Laxm. }\end{array}$ & $\begin{array}{l}\text { Xi Bo Li } \\
\text { Ya Liao }\end{array}$ & Suan liu liu, Mian tiao & Herb & $\begin{array}{l}\text { Stem, } \\
\text { leaf, } \\
\text { flower }\end{array}$ & 0 & 0 & 7 & 43 & 0 & 10 & 60 & 19.05 \\
\hline Polygonaceae & $\begin{array}{l}\text { Rheum palmatum } \\
\mathrm{L} \text {. }\end{array}$ & $\begin{array}{l}\text { Zhang } \\
\text { Ye Da } \\
\text { Huang }\end{array}$ & $\begin{array}{l}\text { Dai huang, Zhang ye da } \\
\text { huang }\end{array}$ & Herb & $\begin{array}{l}\text { Stem, } \\
\text { leaf }\end{array}$ & 0 & 0 & 51 & 49 & 14 & 16 & 130 & 41.27 \\
\hline Polygonaceae & $\begin{array}{l}\text { Rheum tanguticum } \\
\text { Maxim. ex Balf. }\end{array}$ & $\begin{array}{l}\text { Ji Zhua } \\
\text { Da } \\
\text { Huang }\end{array}$ & $\begin{array}{l}\text { Dai huang, Liu pan shan ji } \\
\text { zhau da huang }\end{array}$ & Herb & $\begin{array}{l}\text { Stem, } \\
\text { leaf }\end{array}$ & 0 & 0 & 0 & 0 & 14 & 0 & 14 & 4.44 \\
\hline Polygonaceae & Rumex acetosa $\mathrm{L}$. & $\begin{array}{l}\text { Suan } \\
\text { Mo }\end{array}$ & $\begin{array}{l}\text { Xiao ye suan bu liu liu, } \\
\text { Xiao ye suan mo }\end{array}$ & Herb & $\begin{array}{l}\text { Stem, } \\
\text { leaf }\end{array}$ & 0 & 0 & 0 & 0 & 14 & 0 & 14 & 4.44 \\
\hline Polygonaceae & Rumex crispus L. & $\begin{array}{l}\text { Zhou Ye } \\
\text { Suan } \\
\text { Mo }\end{array}$ & Lv er duo, Tu dai huang & Herb & $\begin{array}{l}\text { Stem, } \\
\text { leaf }\end{array}$ & 0 & 0 & 51 & 49 & 4 & 2 & 106 & 33.65 \\
\hline Polygonaceae & Rumex patientia L. & $\begin{array}{l}\text { Ba Tian } \\
\text { Suan } \\
\text { Mo }\end{array}$ & $\begin{array}{l}\text { Lv er duo guang zi, Lv er } \\
\text { gua }\end{array}$ & Herb & $\begin{array}{l}\text { Stem, } \\
\text { leaf }\end{array}$ & 0 & 0 & 51 & 49 & 4 & 2 & 106 & 33.65 \\
\hline Caryophyllaceae & $\begin{array}{l}\text { Dianthus chinensis } \\
\mathrm{L} \text {. }\end{array}$ & Shi Zhu & Hong chou zi hua & Herb & $\begin{array}{l}\text { Stem, } \\
\text { leaf }\end{array}$ & 0 & 0 & 0 & 0 & 22 & 0 & 22 & 6.98 \\
\hline Caryophyllaceae & $\begin{array}{l}\text { Dianthus superbus } \\
\text { L. }\end{array}$ & Qu Mai & Qu mai & Herb & $\begin{array}{l}\text { Stem, } \\
\text { leaf }\end{array}$ & 0 & 0 & 0 & 0 & 5 & 0 & 5 & 1.59 \\
\hline Caryophyllaceae & $\begin{array}{l}\text { Stellaria dichotoma } \\
\text { var. lanceolata } \\
\text { Bunge }\end{array}$ & $\begin{array}{l}\text { Yin Chai } \\
\mathrm{Hu}\end{array}$ & Yin chai hu & Herb & $\begin{array}{l}\text { Stem, } \\
\text { leaf }\end{array}$ & 0 & 0 & 0 & 0 & 11 & 0 & 11 & 3.49 \\
\hline Amaranthaceae & $\begin{array}{l}\text { Salsola kali subsp. } \\
\text { tragus (L.) Čelak. }\end{array}$ & $\begin{array}{l}\text { Ci Sha } \\
\text { Peng }\end{array}$ & Ci peng & Herb & $\begin{array}{l}\text { Stem, } \\
\text { leaf }\end{array}$ & 30 & 21 & 29 & 30 & 6 & 35 & 151 & 47.94 \\
\hline Amaranthaceae & Salsola collina Pall. & $\begin{array}{l}\text { Zhu } \\
\text { Mao Cai }\end{array}$ & $\begin{array}{l}\text { Ci peng, Zheng yan zi zha } \\
\text { li zi, Peng zi cai }\end{array}$ & Herb & $\begin{array}{l}\text { Stem, } \\
\text { leaf }\end{array}$ & 32 & 18 & 67 & 50 & 23 & 35 & 225 & 71.43 \\
\hline Amaranthaceae & $\begin{array}{l}\text { Salsola passerina } \\
\text { Bunge }\end{array}$ & $\begin{array}{l}\text { Zhen } \\
\text { Zhu } \\
\text { Zhu } \\
\text { Mao Cai }\end{array}$ & Zhen zhu chai, Ha ma tou & Shrub & $\begin{array}{l}\text { Stem, } \\
\text { leaf }\end{array}$ & 0 & 0 & 60 & 49 & 0 & 0 & 109 & 34.6 \\
\hline Amaranthaceae & $\begin{array}{l}\text { Agriophyllum } \\
\text { squarrosum (L.) } \\
\text { Moq. }\end{array}$ & $\begin{array}{l}\text { Sha } \\
\text { Peng }\end{array}$ & Deng suo & Herb & $\begin{array}{l}\text { Stem, } \\
\text { leaf, } \\
\text { seed }\end{array}$ & 0 & 0 & 60 & 49 & 0 & 0 & 109 & 34.6 \\
\hline Amaranthaceae & $\begin{array}{l}\text { Amaranthus } \\
\text { retroflexus } L \text {. }\end{array}$ & $\begin{array}{l}\text { Fan Zhi } \\
\text { Xian }\end{array}$ & Ye ren han, Gan sui gu & Herb & $\begin{array}{l}\text { Stem, } \\
\text { leaf, } \\
\text { flower, } \\
\text { seed }\end{array}$ & 0 & 0 & 1 & 0 & 32 & 0 & 33 & 10.48 \\
\hline Amaranthaceae & $\begin{array}{l}\text { Atriplex } \\
\text { centralasiatica lljin }\end{array}$ & $\begin{array}{l}\text { Zhong } \\
\text { Ya Bin } \\
\text { Li }\end{array}$ & $\begin{array}{l}\text { Yang er duo hui tiao, Ma } \\
\text { luo luo }\end{array}$ & Herb & $\begin{array}{l}\text { Stem, } \\
\text { leaf }\end{array}$ & 0 & 0 & 0 & 0 & 0 & 37 & 37 & 11.75 \\
\hline Amaranthaceae & $\begin{array}{l}\text { Atriplex fera (L.) } \\
\text { Bunge }\end{array}$ & $\begin{array}{l}\text { Ye Bin } \\
\mathrm{Li}\end{array}$ & Yang er duo hui tiao & Herb & $\begin{array}{l}\text { Stem, } \\
\text { leaf }\end{array}$ & 0 & 0 & 0 & 0 & 0 & 36 & 36 & 11.43 \\
\hline Amaranthaceae & Atriplex sibirica L. & $\begin{array}{l}\text { Xi Bo Li } \\
\text { Ya Bin } \\
\text { Li }\end{array}$ & Ma hui tiao & Herb & $\begin{array}{l}\text { Stem, } \\
\text { leaf }\end{array}$ & 0 & 0 & 62 & 56 & 0 & 0 & 118 & 37.46 \\
\hline Amaranthaceae & $\begin{array}{l}\text { Chenopodium } \\
\text { album L. }\end{array}$ & $\mathrm{Li}$ & Hui tiao & Herb & $\begin{array}{l}\text { Stem, } \\
\text { leaf }\end{array}$ & 18 & 12 & 62 & 57 & 33 & 36 & 218 & 69.21 \\
\hline Amaranthaceae & Chenopodium & Hui LV & Hui tiao & Herb & Stem, & 17 & 0 & 62 & 57 & 33 & 36 & 205 & 65.08 \\
\hline
\end{tabular}


Table 4 Traditional forage plants and their relative frequency of citation (RFC) (Continued)

\begin{tabular}{|c|c|c|c|c|c|c|c|c|c|c|c|c|c|}
\hline \multirow[t]{2}{*}{ Family name } & \multirow[t]{2}{*}{ Scientific name } & \multirow[t]{2}{*}{$\begin{array}{l}\text { Chinese } \\
\text { name }\end{array}$} & \multirow[t]{2}{*}{ Local name } & \multirow[t]{2}{*}{$\begin{array}{l}\text { Life } \\
\text { form }\end{array}$} & \multirow[t]{2}{*}{$\begin{array}{l}\text { Part } \\
\text { used }\end{array}$} & \multicolumn{6}{|c|}{$\begin{array}{l}\text { Frequency of citations } \\
\text { in the surveyed villages }\end{array}$} & \multirow[t]{2}{*}{$\begin{array}{l}\text { Frequency } \\
\text { of citation (FC) }\end{array}$} & \multirow{2}{*}{$\begin{array}{l}\text { Relative } \\
\text { frequency } \\
\text { of citation } \\
\text { (RFC) }\end{array}$} \\
\hline & & & & & & a & b & c & $d$ & e & $f$ & & \\
\hline & glaucum L. & $\mathrm{Li}$ & & & leaf & & & & & & & & \\
\hline Amaranthaceae & $\begin{array}{l}\text { Chenopodium } \\
\text { hybridum L. }\end{array}$ & $\begin{array}{l}\text { Za Pei } \\
\mathrm{Li}\end{array}$ & Hui tiao & Herb & $\begin{array}{l}\text { Stem, } \\
\text { leaf }\end{array}$ & 0 & 0 & 0 & 0 & 33 & 0 & 33 & 10.48 \\
\hline Amaranthaceae & $\begin{array}{l}\text { Dysphania } \\
\text { schraderiana } \\
\text { (Schult.) Mosyakin } \\
\text { \& Clemants }\end{array}$ & $\begin{array}{l}\text { Ju Ye } \\
\text { Xiang Li }\end{array}$ & Xiao ye hui tiao & Herb & $\begin{array}{l}\text { Stem, } \\
\text { leaf }\end{array}$ & 0 & 0 & 0 & 0 & 32 & 0 & 32 & 10.16 \\
\hline Amaranthaceae & $\begin{array}{l}\text { Halogeton } \\
\text { arachnoideus }\end{array}$ & $\begin{array}{l}\text { Bai Jing } \\
\text { Yan } \\
\text { Sheng } \\
\text { Cao }\end{array}$ & Shui peng hao, Shui hao & Herb & $\begin{array}{l}\text { Stem, } \\
\text { leaf }\end{array}$ & 0 & 5 & 4 & 49 & 34 & 33 & 125 & 39.68 \\
\hline Amaranthaceae & $\begin{array}{l}\text { Haloxylon } \\
\text { ammodendron } \\
\text { (C.A.Mey.) Bunge } \\
\text { ex Fenzl }\end{array}$ & Suo Suo & Suo suo & $\begin{array}{l}\text { Shrub } \\
\text { or } \\
\text { Tree }\end{array}$ & $\begin{array}{l}\text { Stem, } \\
\text { leaf }\end{array}$ & 0 & 0 & 18 & 43 & 0 & 0 & 61 & 19.37 \\
\hline Amaranthaceae & $\begin{array}{l}\text { Kalidium } \\
\text { cuspidatum (Ung.- } \\
\text { Sternb.) Grubov }\end{array}$ & $\begin{array}{l}\text { Jian Ye } \\
\text { Yan } \\
\text { Zhua } \\
\text { Zhua }\end{array}$ & $\begin{array}{l}\text { Lao shu shi dan dan, Yan } \\
\text { hao }\end{array}$ & Shrub & $\begin{array}{l}\text { Stem, } \\
\text { leaf }\end{array}$ & 0 & 0 & 42 & 43 & 0 & 0 & 85 & 26.98 \\
\hline Amaranthaceae & $\begin{array}{l}\text { Krascheninnikovia } \\
\text { ceratoides (L.) } \\
\text { Gueldenst. }\end{array}$ & $\begin{array}{l}\text { TuO } \\
\text { Rong Li }\end{array}$ & You ruo li & Shrub & $\begin{array}{l}\text { Stem, } \\
\text { leaf, } \\
\text { flower, } \\
\text { fruit }\end{array}$ & 0 & 0 & 29 & 43 & 0 & 0 & 72 & 22.86 \\
\hline Amaranthaceae & $\begin{array}{l}\text { Suaeda glauca } \\
\text { (Bunge) Bunge }\end{array}$ & $\begin{array}{l}\text { Jian } \\
\text { Peng }\end{array}$ & $\begin{array}{l}\text { Lv wei ba yan hao, Yan } \\
\text { hao hao, Jian hao, Jian } \\
\text { peng }\end{array}$ & Herb & $\begin{array}{l}\text { Stem, } \\
\text { leaf }\end{array}$ & 0 & 6 & 60 & 54 & 0 & 34 & 154 & 48.89 \\
\hline Amaranthaceae & $\begin{array}{l}\text { Sympegma regelii } \\
\text { Bunge }\end{array}$ & $\begin{array}{l}\text { He Tou } \\
\text { Cao }\end{array}$ & $\begin{array}{l}\text { Hei chai, He chai, Hei ma } \\
\text { tou chai, He lao gua chai }\end{array}$ & Shrub & $\begin{array}{l}\text { Stem, } \\
\text { leaf }\end{array}$ & 0 & 0 & 42 & 43 & 0 & 0 & 85 & 26.98 \\
\hline Amaranthaceae & $\begin{array}{l}\text { Bassia scoparia (L.) } \\
\text { A.J.Scott }\end{array}$ & Di Fu & $\begin{array}{l}\text { Mao luo li, Li jing, Ye li } \\
\text { jing }\end{array}$ & Herb & $\begin{array}{l}\text { Stem, } \\
\text { leaf }\end{array}$ & 11 & 0 & 42 & 35 & 1 & 11 & 100 & 31.75 \\
\hline Amaranthaceae & $\begin{array}{l}\text { Corispermum } \\
\text { patelliforme }\end{array}$ & $\begin{array}{l}\text { Die Guo } \\
\text { Chong } \\
\text { Shi }\end{array}$ & Mian peng & Herb & $\begin{array}{l}\text { Stem, } \\
\text { leaf, } \\
\text { seed }\end{array}$ & 0 & 17 & 60 & 55 & 0 & 38 & 170 & 53.97 \\
\hline Amaranthaceae & $\begin{array}{l}\text { Corispermum } \\
\text { patelliforme Iljin }\end{array}$ & $\begin{array}{l}\text { Sheng } \\
\text { Chong } \\
\text { Shi }\end{array}$ & Mian peng & Herb & $\begin{array}{l}\text { Stem, } \\
\text { leaf }\end{array}$ & 0 & 17 & 61 & 54 & 0 & 38 & 170 & 53.97 \\
\hline Talinaceae & $\begin{array}{l}\text { Pedicularis } \\
\text { muscicola Maxim. }\end{array}$ & $\begin{array}{l}\text { Xian } \\
\text { Sheng } \\
\text { Ma Xian } \\
\text { Hao }\end{array}$ & Chang chong cao & Herb & $\begin{array}{l}\text { Stem, } \\
\text { leaf }\end{array}$ & 0 & 0 & 0 & 0 & 1 & 0 & 1 & 0.32 \\
\hline Portulacaceae & $\begin{array}{l}\text { Portulaca oleracea } \\
\text { L. }\end{array}$ & $\begin{array}{l}\text { Ma Chi } \\
\text { Xian }\end{array}$ & Pang wa wa cai & Herb & $\begin{array}{l}\text { Stem, } \\
\text { leaf }\end{array}$ & 1 & 5 & 12 & 9 & 19 & 2 & 48 & 15.24 \\
\hline Rubiaceae & Galium aparine L. & $\begin{array}{l}\text { Zhu } \\
\text { Yang } \\
\text { Yang }\end{array}$ & Ran wa zi cao & Herb & $\begin{array}{l}\text { Stem, } \\
\text { leaf }\end{array}$ & 0 & 0 & 0 & 0 & 19 & 0 & 19 & 6.03 \\
\hline Rubiaceae & Galium verum L. & $\begin{array}{l}\text { Peng Zi } \\
\text { Cai }\end{array}$ & Huang mi gan fan & Herb & $\begin{array}{l}\text { Stem, } \\
\text { leaf, } \\
\text { Flower }\end{array}$ & 0 & 0 & 0 & 0 & 1 & 0 & 1 & 0.32 \\
\hline Rubiaceae & Rubia cordifolia L. & $\begin{array}{l}\text { Qian } \\
\text { Cao }\end{array}$ & Qian cao zi, Ran wa zi & Liana & $\begin{array}{l}\text { Stem, } \\
\text { leaf }\end{array}$ & 0 & 0 & 2 & 0 & 3 & 0 & 5 & 1.59 \\
\hline Apocynaceae & $\begin{array}{l}\text { Cynanchum } \\
\text { absconditum Liede }\end{array}$ & $\begin{array}{l}\text { Di Shao } \\
\text { Gua }\end{array}$ & Hao gua zi & Herb & $\begin{array}{l}\text { Leaf, } \\
\text { fruit }\end{array}$ & 0 & 0 & 38 & 20 & 0 & 31 & 89 & 28.25 \\
\hline Apocynaceae & $\begin{array}{l}\text { Cynanchum } \\
\text { acutum L. }\end{array}$ & $\begin{array}{l}\text { Yang } \\
\text { Jiao Zi } \\
\text { Cao }\end{array}$ & $\begin{array}{l}\text { Yang nai jiao jiao, Yang } \\
\text { jiao zi }\end{array}$ & Herb & Leaf & 0 & 0 & 25 & 48 & 0 & 6 & 79 & 25.08 \\
\hline
\end{tabular}


Table 4 Traditional forage plants and their relative frequency of citation (RFC) (Continued)

\begin{tabular}{|c|c|c|c|c|c|c|c|c|c|c|c|c|c|}
\hline \multirow[t]{2}{*}{ Family name } & \multirow[t]{2}{*}{ Scientific name } & \multirow[t]{2}{*}{$\begin{array}{l}\text { Chinese } \\
\text { name }\end{array}$} & \multirow[t]{2}{*}{ Local name } & \multirow[t]{2}{*}{$\begin{array}{l}\text { Life } \\
\text { form }\end{array}$} & \multirow[t]{2}{*}{$\begin{array}{l}\text { Part } \\
\text { used }\end{array}$} & \multicolumn{6}{|c|}{$\begin{array}{l}\text { Frequency of citations } \\
\text { in the surveyed villages }\end{array}$} & \multirow[t]{2}{*}{$\begin{array}{l}\text { Frequency } \\
\text { of citation (FC) }\end{array}$} & \multirow{2}{*}{$\begin{array}{l}\text { Relative } \\
\text { frequency } \\
\text { of citation } \\
\text { (RFC) }\end{array}$} \\
\hline & & & & & & a & $b$ & c & $d$ & e & $f$ & & \\
\hline Apocynaceae & $\begin{array}{l}\text { Cynanchum } \\
\text { chinense R.Br. }\end{array}$ & $\begin{array}{l}\text { E Rong } \\
\text { Teng }\end{array}$ & $\begin{array}{l}\text { Yang nai jiao jiao, Ma hao } \\
\text { gua zi }\end{array}$ & Herb & Leaf & 0 & 1 & 55 & 48 & 0 & 0 & 104 & 33.02 \\
\hline Boraginaceae & $\begin{array}{l}\text { Lappula myosotis } \\
\text { V. Wolf }\end{array}$ & He Shi & Ran ran zi, Mao ran ran & Herb & $\begin{array}{l}\text { Stem, } \\
\text { leaf }\end{array}$ & 0 & 4 & 58 & 58 & 0 & 30 & 150 & 47.62 \\
\hline Boraginaceae & $\begin{array}{l}\text { Lappula squarrosa } \\
\text { (Retz.) Dumort. }\end{array}$ & $\begin{array}{l}\text { Lan Ci } \\
\text { He Shi }\end{array}$ & Zhan sheng cao & Herb & $\begin{array}{l}\text { Stem, } \\
\text { leaf }\end{array}$ & 0 & 0 & 0 & 0 & 13 & 0 & 13 & 4.13 \\
\hline Boraginaceae & $\begin{array}{l}\text { Lappula squarrosa } \\
\text { subsp. } \\
\text { heteracantha } \\
\text { (Ledeb.) Chater }\end{array}$ & $\begin{array}{l}\text { Yi Ci He } \\
\text { Shi }\end{array}$ & Ran ran zi, Mao ran ran & Herb & $\begin{array}{l}\text { Stem, } \\
\text { leaf }\end{array}$ & 0 & 6 & 64 & 58 & 0 & 30 & 158 & 50.16 \\
\hline Convolvulaceae & $\begin{array}{l}\text { Calystegia } \\
\text { hederacea Wall. }\end{array}$ & $\begin{array}{l}\text { Da Wan } \\
\text { Hua }\end{array}$ & $\begin{array}{l}\text { Fu zi miao, Tian xuan hua, } \\
\text { Gu zi man, Ku zi man, La } \\
\text { ba hua, Da da wai, Bai } \\
\text { hua gu zi man }\end{array}$ & Herb & $\begin{array}{l}\text { Stem, } \\
\text { leaf, } \\
\text { flower }\end{array}$ & 3 & 7 & 1 & 56 & 30 & 35 & 132 & 41.9 \\
\hline Convolvulaceae & $\begin{array}{l}\text { Convolvulus } \\
\text { arvensis } \mathrm{L} \text {. }\end{array}$ & $\begin{array}{l}\text { Tian } \\
\text { Xuan } \\
\text { Hua }\end{array}$ & $\begin{array}{l}\text { Gu zi man, Ku zi man, Fen } \\
\text { hua gu zi man }\end{array}$ & Herb & $\begin{array}{l}\text { Stem, } \\
\text { leaf, } \\
\text { flower }\end{array}$ & 3 & 7 & 55 & 39 & 2 & 35 & 141 & 44.76 \\
\hline Convolvulaceae & $\begin{array}{l}\text { Convolvulus } \\
\text { tragacanthoides } \\
\text { Turcz. }\end{array}$ & $\begin{array}{l}\text { Ci Xuan } \\
\text { Hua }\end{array}$ & Ying zhua ci, Tie dan dan & Shrub & $\begin{array}{l}\text { Stem, } \\
\text { leaf, } \\
\text { flower }\end{array}$ & 0 & 0 & 62 & 39 & 0 & 0 & 101 & 32.06 \\
\hline Convolvulaceae & $\begin{array}{l}\text { Cuscuta chinensis } \\
\text { Lam. }\end{array}$ & Tu Si Zi & Huang tang, Huang chan & Herb & $\begin{array}{l}\text { Stem, } \\
\text { leaf }\end{array}$ & 0 & 0 & 11 & 41 & 0 & 0 & 52 & 16.51 \\
\hline Convolvulaceae & $\begin{array}{l}\text { Ipomoea purpurea } \\
\text { (L.) Roth }\end{array}$ & $\begin{array}{l}\text { Yuan Ye } \\
\text { Qian } \\
\text { Niu }\end{array}$ & $\begin{array}{l}\text { Qian niu hua, La ba hua, } \\
\text { Hei bai chou }\end{array}$ & Herb & $\begin{array}{l}\text { Stem, } \\
\text { leaf, } \\
\text { flower }\end{array}$ & 4 & 3 & 21 & 4 & 3 & 22 & 57 & 18.1 \\
\hline Solanaceae & $\begin{array}{l}\text { Lycium barbarum } \\
\text { L. }\end{array}$ & $\begin{array}{l}\text { Ning } \\
\text { Xia Gou } \\
\text { Qi }\end{array}$ & Gou ji zi, Gou qi zi & Shrub & $\begin{array}{l}\text { Stem, } \\
\text { leaf }\end{array}$ & 0 & 0 & 15 & 1 & 0 & 6 & 22 & 6.98 \\
\hline Solanaceae & $\begin{array}{l}\text { Lycium chinense } \\
\text { Mill. }\end{array}$ & Gou Qi & Gou ji zi, Gou qi zi & Shrub & $\begin{array}{l}\text { Stem, } \\
\text { leaf }\end{array}$ & 0 & 0 & 15 & 1 & 0 & 6 & 22 & 6.98 \\
\hline Solanaceae & $\begin{array}{l}\text { Solanum } \\
\text { americanum Mill. }\end{array}$ & $\begin{array}{l}\text { Long } \\
\text { Kui }\end{array}$ & Ye wei hua, Ye hua hua & Herb & $\begin{array}{l}\text { Stem, } \\
\text { leaf }\end{array}$ & 0 & 0 & 0 & 0 & 12 & 0 & 12 & 3.81 \\
\hline Solanaceae & $\begin{array}{l}\text { Solanum } \\
\text { tuberosum L. }\end{array}$ & $\begin{array}{l}\text { Ma Ling } \\
\text { Shu }\end{array}$ & Yang yu & Herb & $\begin{array}{l}\text { Stem, } \\
\text { leaf }\end{array}$ & 0 & 0 & 0 & 0 & 0 & 4 & 4 & 1.27 \\
\hline Plantaginaceae & Plantago asiatica L. & $\begin{array}{l}\text { Che } \\
\text { Qian }\end{array}$ & $\begin{array}{l}\text { Che gian cao, Niu she tou, } \\
\text { Niu er duo }\end{array}$ & Herb & $\begin{array}{l}\text { Stem, } \\
\text { leaf, } \\
\text { flower, } \\
\text { fruit }\end{array}$ & 7 & 2 & 0 & 0 & 28 & 14 & 51 & 16.19 \\
\hline Plantaginaceae & $\begin{array}{l}\text { Plantago depressa } \\
\text { Willd. }\end{array}$ & $\begin{array}{l}\text { Ping } \\
\text { Che } \\
\text { Qian }\end{array}$ & $\begin{array}{l}\text { Che gian cao, Niu she tou, } \\
\text { Niu er duo }\end{array}$ & Herb & $\begin{array}{l}\text { Stem, } \\
\text { leaf }\end{array}$ & 7 & 2 & 0 & 0 & 28 & 14 & 51 & 16.19 \\
\hline Plantaginaceae & Plantago major L. & $\begin{array}{l}\text { Da Che } \\
\text { Qian }\end{array}$ & $\begin{array}{l}\text { Che qian cao, Niu she tou, } \\
\text { Niu er duo }\end{array}$ & Herb & $\begin{array}{l}\text { Stem, } \\
\text { leaf, } \\
\text { flower, } \\
\text { fruit }\end{array}$ & 7 & 2 & 0 & 0 & 28 & 14 & 51 & 16.19 \\
\hline Lamiaceae & $\begin{array}{l}\text { Mentha canadensis } \\
\mathrm{L} \text {. }\end{array}$ & $\mathrm{Bo} \mathrm{He}$ & Ye bo he & Herb & $\begin{array}{l}\text { Stem, } \\
\text { leaf }\end{array}$ & 0 & 0 & 0 & 0 & 1 & 0 & 1 & 0.32 \\
\hline Lamiaceae & $\begin{array}{l}\text { Lagochilus ilicifolius } \\
\text { Bunge ex Benth. }\end{array}$ & $\begin{array}{l}\text { Dong } \\
\text { Qing Ye } \\
\text { Tu } \\
\text { Chun } \\
\text { Hua }\end{array}$ & Ji guan zi & Herb & $\begin{array}{l}\text { Stem, } \\
\text { leaf }\end{array}$ & 0 & 0 & 1 & 23 & 0 & 0 & 24 & 7.62 \\
\hline Lamiaceae & $\begin{array}{l}\text { Dracocephalum } \\
\text { heterophyllum } \\
\text { Benth. }\end{array}$ & $\begin{array}{l}\text { Bai Hua } \\
\text { Zhi Zi } \\
\text { Hua }\end{array}$ & Mi guan guan & Herb & $\begin{array}{l}\text { Stem, } \\
\text { leaf }\end{array}$ & 0 & 0 & 0 & 0 & 1 & 10 & 11 & 3.49 \\
\hline
\end{tabular}


Table 4 Traditional forage plants and their relative frequency of citation (RFC) (Continued)

\begin{tabular}{|c|c|c|c|c|c|c|c|c|c|c|c|c|c|}
\hline \multirow[t]{2}{*}{ Family name } & \multirow[t]{2}{*}{ Scientific name } & \multirow[t]{2}{*}{$\begin{array}{l}\text { Chinese } \\
\text { name }\end{array}$} & \multirow[t]{2}{*}{ Local name } & \multirow[t]{2}{*}{$\begin{array}{l}\text { Life } \\
\text { form }\end{array}$} & \multirow[t]{2}{*}{$\begin{array}{l}\text { Part } \\
\text { used }\end{array}$} & \multicolumn{6}{|c|}{$\begin{array}{l}\text { Frequency of citations } \\
\text { in the surveyed villages }\end{array}$} & \multirow{2}{*}{$\begin{array}{l}\text { Frequency } \\
\text { of citation (FC) }\end{array}$} & \multirow{2}{*}{$\begin{array}{l}\text { Relative } \\
\text { frequency } \\
\text { of citation } \\
\text { (RFC) }\end{array}$} \\
\hline & & & & & & $\mathrm{a}$ & $b$ & c & $d$ & e & $f$ & & \\
\hline Lamiaceae & $\begin{array}{l}\text { Leonurus japonicus } \\
\text { Houtt. }\end{array}$ & $\begin{array}{l}\text { Yi Mu } \\
\mathrm{CaO}\end{array}$ & Jie jie hao & Herb & $\begin{array}{l}\text { Stem, } \\
\text { leaf }\end{array}$ & 0 & 0 & 18 & 10 & 0 & 0 & 28 & 8.89 \\
\hline Lamiaceae & $\begin{array}{l}\text { Stachys affinis } \\
\text { Bunge }\end{array}$ & $\begin{array}{l}\text { Gan Lu } \\
\mathrm{Zi}\end{array}$ & Di liu zi & Herb & $\begin{array}{l}\text { Stem, } \\
\text { leaf }\end{array}$ & 0 & 2 & 6 & 2 & 1 & 0 & 11 & 3.49 \\
\hline Lamiaceae & $\begin{array}{l}\text { Thymus } \\
\text { mongolicus } \\
\text { (Ronniger) } \\
\text { Ronniger }\end{array}$ & $\begin{array}{l}\text { Bai Li } \\
\text { Xiang }\end{array}$ & Di jiao & Shrub & $\begin{array}{l}\text { Stem, } \\
\text { leaf }\end{array}$ & 0 & 0 & 0 & 0 & 20 & 1 & 21 & 6.67 \\
\hline Campanulaceae & $\begin{array}{l}\text { Adenophora } \\
\text { potaninii Korsh. }\end{array}$ & $\begin{array}{l}\text { Pao Sha } \\
\text { Shen }\end{array}$ & Niu ling hua & Herb & $\begin{array}{l}\text { Stem, } \\
\text { leaf }\end{array}$ & 0 & 0 & 0 & 0 & 17 & 0 & 17 & 5.4 \\
\hline Campanulaceae & $\begin{array}{l}\text { Codonopsis pilosula } \\
\text { (Franch.) Nannf. }\end{array}$ & $\begin{array}{l}\text { Dang } \\
\text { Shen }\end{array}$ & Dang sen & Herb & $\begin{array}{l}\text { Stem, } \\
\text { leaf }\end{array}$ & 0 & 0 & 0 & 0 & 19 & 0 & 19 & 6.03 \\
\hline Compositae & $\begin{array}{l}\text { Ajania achilleoides } \\
\text { (Turcz.) Poljakov ex } \\
\text { Grubov }\end{array}$ & $\begin{array}{l}\text { Shi } \\
\text { Zhuang } \\
\text { Ya Ju }\end{array}$ & Ga ji hao, bia mi hao & Shrub & Leaf & 0 & 0 & 29 & 42 & 0 & 0 & 71 & 22.54 \\
\hline Compositae & $\begin{array}{l}\text { Ajania fruticulosa } \\
\text { (Ledeb.) Poljakov }\end{array}$ & $\begin{array}{l}\text { Guan } \\
\text { Mu Ya } \\
\text { Ju }\end{array}$ & Ga ji hao & Shrub & Leaf & 0 & 0 & 29 & 32 & 0 & 0 & 61 & 19.37 \\
\hline Compositae & Arctium lappa L. & $\begin{array}{l}\text { Niu } \\
\text { Pang }\end{array}$ & Niu zi, Da li zi & Herb & $\begin{array}{l}\text { Stem, } \\
\text { leaf }\end{array}$ & 0 & 0 & 0 & 0 & 1 & 0 & 1 & 0.32 \\
\hline Compositae & Artemisia annua L. & $\begin{array}{l}\text { Huang } \\
\text { Hua } \\
\text { HaO }\end{array}$ & Chou hao, Huang hao & Herb & $\begin{array}{l}\text { Stem, } \\
\text { leaf }\end{array}$ & 0 & 0 & 29 & 31 & 30 & 27 & 117 & 37.14 \\
\hline Compositae & $\begin{array}{l}\text { Artemisia argyi } \\
\text { H.Lév. \& Vaniot }\end{array}$ & $\mathrm{Ai}$ & Nai, Ai & Herb & Flower & 5 & 5 & 10 & 2 & 27 & 29 & 78 & 24.76 \\
\hline Compositae & $\begin{array}{l}\text { Artemisia } \\
\text { blepharolepis } \\
\text { Bunge }\end{array}$ & $\begin{array}{l}\text { Bai Sha } \\
\text { Hao }\end{array}$ & bia sha hao & Shrub & $\begin{array}{l}\text { Stem, } \\
\text { leaf }\end{array}$ & 4 & 5 & 51 & 54 & 0 & 27 & 141 & 44.76 \\
\hline Compositae & $\begin{array}{l}\text { Artemisia capillaris } \\
\text { Thunb. }\end{array}$ & $\begin{array}{l}\text { Yin } \\
\text { Chen } \\
\text { Hao }\end{array}$ & Bai hao tou zi, You hao & $\begin{array}{l}\text { Herb } \\
\text { or } \\
\text { Shrub }\end{array}$ & $\begin{array}{l}\text { Stem, } \\
\text { leaf }\end{array}$ & 18 & 21 & 61 & 49 & 34 & 30 & 213 & 67.62 \\
\hline Compositae & $\begin{array}{l}\text { Artemisia } \\
\text { desertorum Spreng. }\end{array}$ & $\begin{array}{l}\text { Hei Sha } \\
\text { Hao }\end{array}$ & Sha hao, You hao & Shrub & $\begin{array}{l}\text { Stem, } \\
\text { leaf }\end{array}$ & 4 & 16 & 52 & 55 & 0 & 27 & 154 & 48.89 \\
\hline Compositae & $\begin{array}{l}\text { Artemisia dubia L. } \\
\text { ex B.D.Jacks. }\end{array}$ & $\begin{array}{l}\text { Wu Mao } \\
\text { Niu Wei } \\
\text { Hao }\end{array}$ & Yi zi hao & Herb & Leaf & 0 & 0 & 51 & 0 & 0 & 0 & 51 & 16.19 \\
\hline Compositae & $\begin{array}{l}\text { Artemisia frigida } \\
\text { Willd. }\end{array}$ & Len Hao & Chuan di hao & Herb & $\begin{array}{l}\text { Stem, } \\
\text { leaf }\end{array}$ & 0 & 0 & 29 & 31 & 0 & 0 & 60 & 19.05 \\
\hline Compositae & $\begin{array}{l}\text { Artemisia giraldii } \\
\text { Pamp. }\end{array}$ & $\begin{array}{l}\text { Hua Bei } \\
\text { Mi Hao }\end{array}$ & Jiao hao & Herb & $\begin{array}{l}\text { Stem, } \\
\text { leaf, } \\
\text { fruit }\end{array}$ & 0 & 0 & 51 & 38 & 0 & 0 & 89 & 28.25 \\
\hline Compositae & $\begin{array}{l}\text { Artemisia gmelinii } \\
\text { Weber ex Stechm. }\end{array}$ & $\begin{array}{l}\text { Bai Lian } \\
\text { Hao }\end{array}$ & $\begin{array}{l}\text { A ji hao, Dai mi hao, Ying } \\
\text { gan gan hao, Tie gan hao }\end{array}$ & Herb & $\begin{array}{l}\text { Stem, } \\
\text { leaf }\end{array}$ & 0 & 0 & 29 & 31 & 18 & 24 & 102 & 32.38 \\
\hline Compositae & $\begin{array}{l}\text { Artemisia scoparia } \\
\text { Waldst. \& Kitam. }\end{array}$ & $\begin{array}{l}\text { Zhu } \\
\text { Mao } \\
\text { Hao }\end{array}$ & Bai hao tou zi , You hao & Herb & $\begin{array}{l}\text { Stem, } \\
\text { leaf }\end{array}$ & 18 & 21 & 62 & 59 & 34 & 36 & 230 & 73.02 \\
\hline Compositae & $\begin{array}{l}\text { Carduus crispus } \\
\text { Guirão ex Nyman }\end{array}$ & $\begin{array}{l}\text { Si Mao } \\
\text { Fei Lian }\end{array}$ & Ci gai & Herb & $\begin{array}{l}\text { Leaf, } \\
\text { flower }\end{array}$ & 0 & 4 & 0 & 0 & 0 & 25 & 29 & 9.21 \\
\hline Compositae & $\begin{array}{l}\text { Cirsium arvense (L.) } \\
\text { Scop. }\end{array}$ & Ci Er Cai & $\begin{array}{l}\text { Ma ci ji, Ci ji gai, Xiao ji, } \\
\text { Da ji }\end{array}$ & Herb & $\begin{array}{l}\text { Leaf, } \\
\text { flower }\end{array}$ & 0 & 4 & 25 & 18 & 0 & 25 & 72 & 22.86 \\
\hline Compositae & $\begin{array}{l}\text { Ixeris chinensis } \\
\text { (Thunb. ex Thunb.) } \\
\text { Nakai }\end{array}$ & $\begin{array}{l}\text { Zhong } \\
\text { Hua } \\
\text { Xiao Ku } \\
\text { Mai }\end{array}$ & $\begin{array}{l}\text { Yan ji ji cao, Gua la ji cao, } \\
\text { Ma yan wo cao }\end{array}$ & Herb & $\begin{array}{l}\text { Stem, } \\
\text { leaf }\end{array}$ & 0 & 8 & 53 & 45 & 0 & 28 & 134 & 42.54 \\
\hline
\end{tabular}


Table 4 Traditional forage plants and their relative frequency of citation (RFC) (Continued)

\begin{tabular}{|c|c|c|c|c|c|c|c|c|c|c|c|c|c|}
\hline \multirow[t]{2}{*}{ Family name } & \multirow[t]{2}{*}{ Scientific name } & \multirow[t]{2}{*}{$\begin{array}{l}\text { Chinese } \\
\text { name }\end{array}$} & \multirow[t]{2}{*}{ Local name } & \multirow[t]{2}{*}{$\begin{array}{l}\text { Life } \\
\text { form }\end{array}$} & \multirow[t]{2}{*}{$\begin{array}{l}\text { Part } \\
\text { used }\end{array}$} & \multicolumn{6}{|c|}{$\begin{array}{l}\text { Frequency of citations } \\
\text { in the surveyed villages }\end{array}$} & \multirow[t]{2}{*}{$\begin{array}{l}\text { Frequency } \\
\text { of citation (FC) }\end{array}$} & \multirow{2}{*}{$\begin{array}{l}\text { Relative } \\
\text { frequency } \\
\text { of citation } \\
\text { (RFC) }\end{array}$} \\
\hline & & & & & & a & $b$ & c & $d$ & e & $f$ & & \\
\hline Compositae & $\begin{array}{l}\text { Helianthus annuus } \\
\mathrm{L} \text {. }\end{array}$ & $\begin{array}{l}\text { Xiang Ri } \\
\text { Kui }\end{array}$ & Xiang ri kui & Herb & $\begin{array}{l}\text { Stem, } \\
\text { leaf }\end{array}$ & 11 & 5 & 35 & 36 & 7 & 30 & 124 & 39.37 \\
\hline Compositae & $\begin{array}{l}\text { Helianthus } \\
\text { tuberosus L. }\end{array}$ & Ju Yu & Yang jiang & Herb & $\begin{array}{l}\text { Stem, } \\
\text { leaf, } \\
\text { flower }\end{array}$ & 0 & 0 & 52 & 2 & 0 & 0 & 54 & 17.14 \\
\hline Compositae & $\begin{array}{l}\text { Heteropappus } \\
\text { altaicus (Willd.) } \\
\text { Novopokr. }\end{array}$ & $\begin{array}{l}\text { A Er Tai } \\
\text { Gou Wa } \\
\text { Hua }\end{array}$ & Bai hua cao & Herb & $\begin{array}{l}\text { Stem, } \\
\text { leaf, } \\
\text { flower }\end{array}$ & 0 & 0 & 29 & 33 & 0 & 28 & 90 & 28.57 \\
\hline Compositae & $\begin{array}{l}\text { Lactuca tatarica } \\
\text { (L.) C.A.Mey. }\end{array}$ & Ru Ju & Ma ku ku cai & Herb & $\begin{array}{l}\text { Stem, } \\
\text { leaf }\end{array}$ & 12 & 0 & 54 & 55 & 0 & 45 & 166 & 52.7 \\
\hline Compositae & $\begin{array}{l}\text { Neopallasia } \\
\text { pectinata (Pall.) } \\
\text { Poljakov }\end{array}$ & $\begin{array}{l}\text { Zhi Ye } \\
\text { Hao }\end{array}$ & Mi hao, Mei hao & Herb & $\begin{array}{l}\text { Stem, } \\
\text { leaf }\end{array}$ & 0 & 0 & 29 & 43 & 0 & 22 & 94 & 29.84 \\
\hline Compositae & $\begin{array}{l}\text { Rhaponticum } \\
\text { repens (L.) Hidalgo }\end{array}$ & $\begin{array}{l}\text { Ding Yu } \\
\text { Ju }\end{array}$ & Ku hao & Herb & $\begin{array}{l}\text { Leaf, } \\
\text { flower }\end{array}$ & 0 & 3 & 18 & 29 & 0 & 33 & 83 & 26.35 \\
\hline Compositae & $\begin{array}{l}\text { Saussurea alata } \\
\text { DC. }\end{array}$ & $\begin{array}{l}\text { Yi Jing } \\
\text { Feng } \\
\text { Mao Ju }\end{array}$ & Ye da dou & Herb & $\begin{array}{l}\text { Stem, } \\
\text { leaf }\end{array}$ & 0 & 0 & 0 & 0 & 12 & 0 & 12 & 3.81 \\
\hline Compositae & $\begin{array}{l}\text { Scorzonera } \\
\text { divaricata Turcz. }\end{array}$ & $\begin{array}{l}\text { Cha Zhi } \\
\text { Ya } \\
\text { Cong }\end{array}$ & Nai gua zi & Herb & $\begin{array}{l}\text { Stem, } \\
\text { leaf }\end{array}$ & 0 & 19 & 10 & 33 & 0 & 31 & 93 & 29.52 \\
\hline Compositae & Sonchus arvensis L. & $\begin{array}{l}\text { Ju Mai } \\
\text { Cai }\end{array}$ & $\begin{array}{l}\text { Ku ku cai, Ku xu, Tian ku } \\
\text { ku cai }\end{array}$ & Herb & $\begin{array}{l}\text { Stem, } \\
\text { leaf }\end{array}$ & 17 & 6 & 54 & 55 & 0 & 45 & 177 & 56.19 \\
\hline Compositae & $\begin{array}{l}\text { Sonchus oleraceus } \\
\text { (L.) L. }\end{array}$ & $\begin{array}{l}\mathrm{Ku} \mathrm{Ju} \\
\mathrm{Cai}\end{array}$ & Ku ku cai, Tian ku cai & Herb & $\begin{array}{l}\text { Stem, } \\
\text { leaf }\end{array}$ & 17 & 6 & 54 & 55 & 0 & 45 & 177 & 56.19 \\
\hline Compositae & $\begin{array}{l}\text { Taraxacum } \\
\text { mongolicum } \\
\text { Hand.-Mazz. }\end{array}$ & $\begin{array}{l}\text { Pu } \\
\text { Gong } \\
\text { Ying }\end{array}$ & $\begin{array}{l}\text { Huang huang zi, Huan } \\
\text { huan tai }\end{array}$ & Herb & $\begin{array}{l}\text { Stem, } \\
\text { leaf }\end{array}$ & 18 & 4 & 54 & 0 & 0 & 39 & 115 & 36.51 \\
\hline Compositae & Tussilago farfara L. & $\begin{array}{l}\text { Kuan } \\
\text { Dong }\end{array}$ & Dong hua & Herb & $\begin{array}{l}\text { Stem, } \\
\text { leaf }\end{array}$ & 0 & 0 & 0 & 0 & 1 & 0 & 1 & 0.32 \\
\hline Compositae & $\begin{array}{l}\text { Xanthium } \\
\text { strumarium subsp. } \\
\text { sibiricum (Patrin ex } \\
\text { Widder) Greuter }\end{array}$ & Cang Er & Cang er zi, Cao er & Herb & $\begin{array}{l}\text { Stem, } \\
\text { leaf }\end{array}$ & 0 & 2 & 13 & 20 & 0 & 21 & 56 & 17.78 \\
\hline Apiaceae & $\begin{array}{l}\text { Ligusticum striatum } \\
\text { DC. }\end{array}$ & $\begin{array}{l}\text { Chuan } \\
\text { Xiong }\end{array}$ & Chuan xiong & Herb & $\begin{array}{l}\text { Stem, } \\
\text { leaf }\end{array}$ & 0 & 0 & 0 & 0 & 1 & 0 & 1 & 0.32 \\
\hline Apiaceae & $\begin{array}{l}\text { Anthriscus sylvestris } \\
\text { (L.) Hoffm. }\end{array}$ & E Shen & Ye hong luo bo & Herb & $\begin{array}{l}\text { Stem, } \\
\text { leaf }\end{array}$ & 0 & 0 & 0 & 0 & 15 & 0 & 15 & 4.76 \\
\hline Apiaceae & $\begin{array}{l}\text { Bupleurum } \\
\text { chinense DC. }\end{array}$ & $\begin{array}{l}\text { Bei Chai } \\
\mathrm{Hu}\end{array}$ & Da er duo chai hu & Herb & $\begin{array}{l}\text { Stem, } \\
\text { leaf }\end{array}$ & 0 & 0 & 0 & 0 & 18 & 0 & 18 & 5.71 \\
\hline Apiaceae & Daucus carota $\mathrm{L}$. & $\begin{array}{l}\mathrm{Hu} \text { Luo } \\
\text { Bo }\end{array}$ & Hu luo bo & Herb & Root & 0 & 0 & 25 & 12 & 0 & 0 & 37 & 11.75 \\
\hline Apiaceae & $\begin{array}{l}\text { Ferula bungeana } \\
\text { Kitag. }\end{array}$ & $\begin{array}{l}\text { Sha Hui } \\
\text { Xiang }\end{array}$ & Mian diao diao & Herb & Leaf & 0 & 0 & 0 & 0 & 0 & 4 & 4 & 1.27 \\
\hline Apiaceae & $\begin{array}{l}\text { Ligusticum sinense } \\
\text { Oliv. }\end{array}$ & Gao Ben & Ye chuan xiong & Herb & $\begin{array}{l}\text { Stem, } \\
\text { leaf }\end{array}$ & 0 & 0 & 0 & 0 & 3 & 0 & 3 & 0.95 \\
\hline Apiaceae & $\begin{array}{l}\text { Saposhnikovia } \\
\text { divaricata (Turcz.) } \\
\text { Schischk. }\end{array}$ & $\begin{array}{l}\text { Fang } \\
\text { Feng }\end{array}$ & Han sa jiao, Ma yin zi & Herb & $\begin{array}{l}\text { Stem, } \\
\text { leaf }\end{array}$ & 0 & 0 & 0 & 0 & 6 & 0 & 6 & 1.9 \\
\hline Apiaceae & $\begin{array}{l}\text { Torilis japonica } \\
\text { (Houtt.) DC. }\end{array}$ & $\begin{array}{l}\text { Xiao Qie } \\
\text { Yi }\end{array}$ & $\begin{array}{l}\text { Ye hong luo bo, Ye hui } \\
\text { xiang }\end{array}$ & Herb & Leaf & 0 & 0 & 0 & 0 & 34 & 0 & 34 & 10.79 \\
\hline Ephedraceae & $\begin{array}{l}\text { Ephedra intermedia } \\
\text { Schrenk \& C.A.Mey. }\end{array}$ & $\begin{array}{l}\text { Zhong } \\
\text { Ma } \\
\text { Huang }\end{array}$ & Ma huang & Shrub & $\begin{array}{l}\text { Stem, } \\
\text { leaf }\end{array}$ & 0 & 0 & 51 & 49 & 0 & 17 & 117 & 37.14 \\
\hline
\end{tabular}


Table 4 Traditional forage plants and their relative frequency of citation (RFC) (Continued)

\begin{tabular}{|c|c|c|c|c|c|c|c|c|c|c|c|c|c|}
\hline \multirow[t]{2}{*}{ Family name } & \multirow[t]{2}{*}{ Scientific name } & \multirow[t]{2}{*}{$\begin{array}{l}\text { Chinese } \\
\text { name }\end{array}$} & \multirow[t]{2}{*}{ Local name } & \multirow[t]{2}{*}{$\begin{array}{l}\text { Life } \\
\text { form }\end{array}$} & \multirow[t]{2}{*}{$\begin{array}{l}\text { Part } \\
\text { used }\end{array}$} & \multicolumn{6}{|c|}{$\begin{array}{l}\text { Frequency of citations } \\
\text { in the surveyed villages }\end{array}$} & \multirow[t]{2}{*}{$\begin{array}{l}\text { Frequency } \\
\text { of citation (FC) }\end{array}$} & \multirow{2}{*}{$\begin{array}{l}\text { Relative } \\
\text { frequency } \\
\text { of citation } \\
\text { (RFC) }\end{array}$} \\
\hline & & & & & & a & $\mathrm{b}$ & c & $d$ & e & $f$ & & \\
\hline Equisetaceae & $\begin{array}{l}\text { Equisetum arvense } \\
\mathrm{L} \text {. }\end{array}$ & $\begin{array}{l}\text { Wen } \\
\text { Jing }\end{array}$ & Tuan xu, Duan xu & Herb & Leaf & 0 & 0 & 0 & 0 & 6 & 0 & 6 & 1.9 \\
\hline Equisetaceae & $\begin{array}{l}\text { Equisetum } \\
\text { ramosissimum } \\
\text { Desf. }\end{array}$ & $\begin{array}{l}\text { Jie Jie } \\
\text { Cao }\end{array}$ & Jie jie cao, Jie jie tiao & Herb & Leaf & 0 & 0 & 0 & 0 & 6 & 0 & 6 & 1.9 \\
\hline
\end{tabular}

a great impact on the diversity of traditional knowledge regarding forage plants. Contrarily, Villages $\mathrm{c}$ and $\mathrm{d}$, whose inhabitants migrated less than $2 \mathrm{~km}$, experienced little change in the ecological environment and natural resources, and the short-distance relocation had a nonsignificant impact on the diversity of and knowledge regarding forage plants, as evidenced by the relatively small change in the number of traditional forage plant species used by villagers.

In this study, the JI was used to represent the similarities in traditional forage plants among the six villages (Fig. 3). The higher the $\mathrm{JI}$ is, the greater the similarity in forage plant utilization between two villages. Village a has a similarity of 46.03 to Village b, higher than that to Village e nearby its emigration location, indicating that the utilization of forage plants by Village a is highly similar to the village nearby its immigration location but largely different from its emigration location. Village $b$ shows a high similarity to Village a (46.03) and to Village $\mathrm{f}$ (40.50), indicating that the villagers in Villages $b$ adapted themselves to the new environment through knowledge exchange and fusion with the residents in the immigration area while maintaining a certain homology with Village $f$, from where they moved. This might be attributed to the similarity in plant species between the loess hilly area from where Village $b$ originated and its current location in the semiarid desertified area. In addition to a similarity in the number of forage plant species, Villages $c$ and d show a high similarity (91.73) in the types of forage plant species, indicating that the two villages are highly similar in the use of forage plants because of a close historiogeographical relationship and similar ecological environment and natural resources.

Figure 4 presents a cluster tree for the similarity of forage plant species among the six villages. In the first cluster, Village b shows the closest relationship with Village a, followed by its relationship with Village $f$, fully proving that there are certain homologies in the utilization of forage plants between the immigration and emigration areas. Village a has low similarity to and a large distance function with respect to Village e, near where it originated, which might be related to the large difference in natural resources between the two villages. In the second cluster, Villages $c$ and $d$ show the closest relationship in terms of forage plant utilization, suggesting that the two villages have high similarity in forage plant diversity and related knowledge after a short-distance migration within the same ecological type. This finding is consistent with the results from the JI analysis between the villages. Our findings were consistent among different analysis methods.

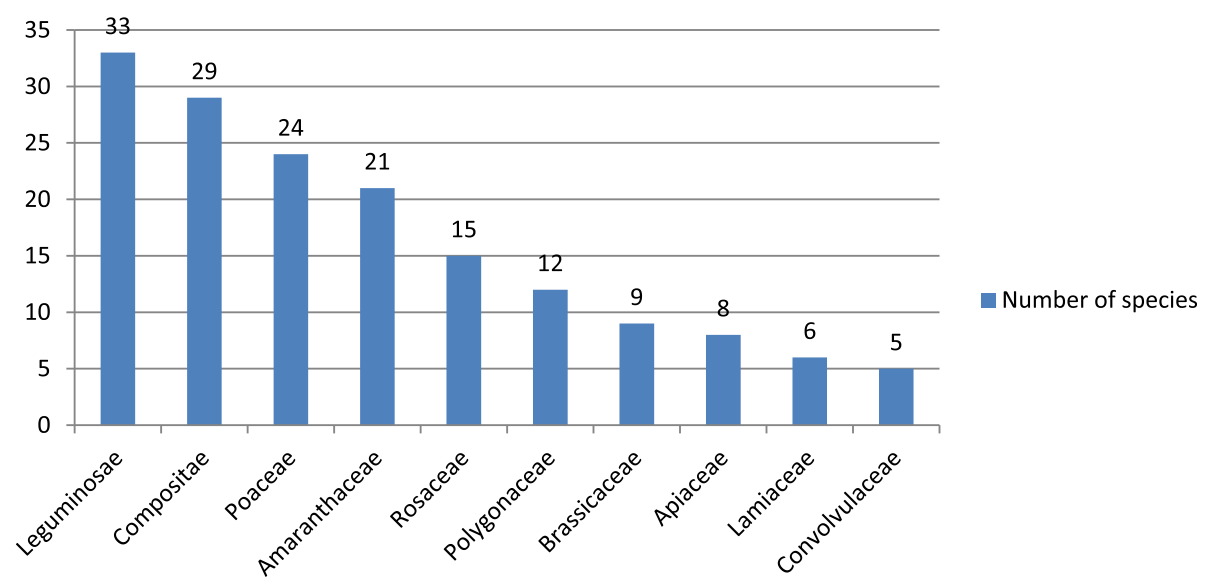

Fig. 2 Plant families that include at least five forage plant species in the research area 


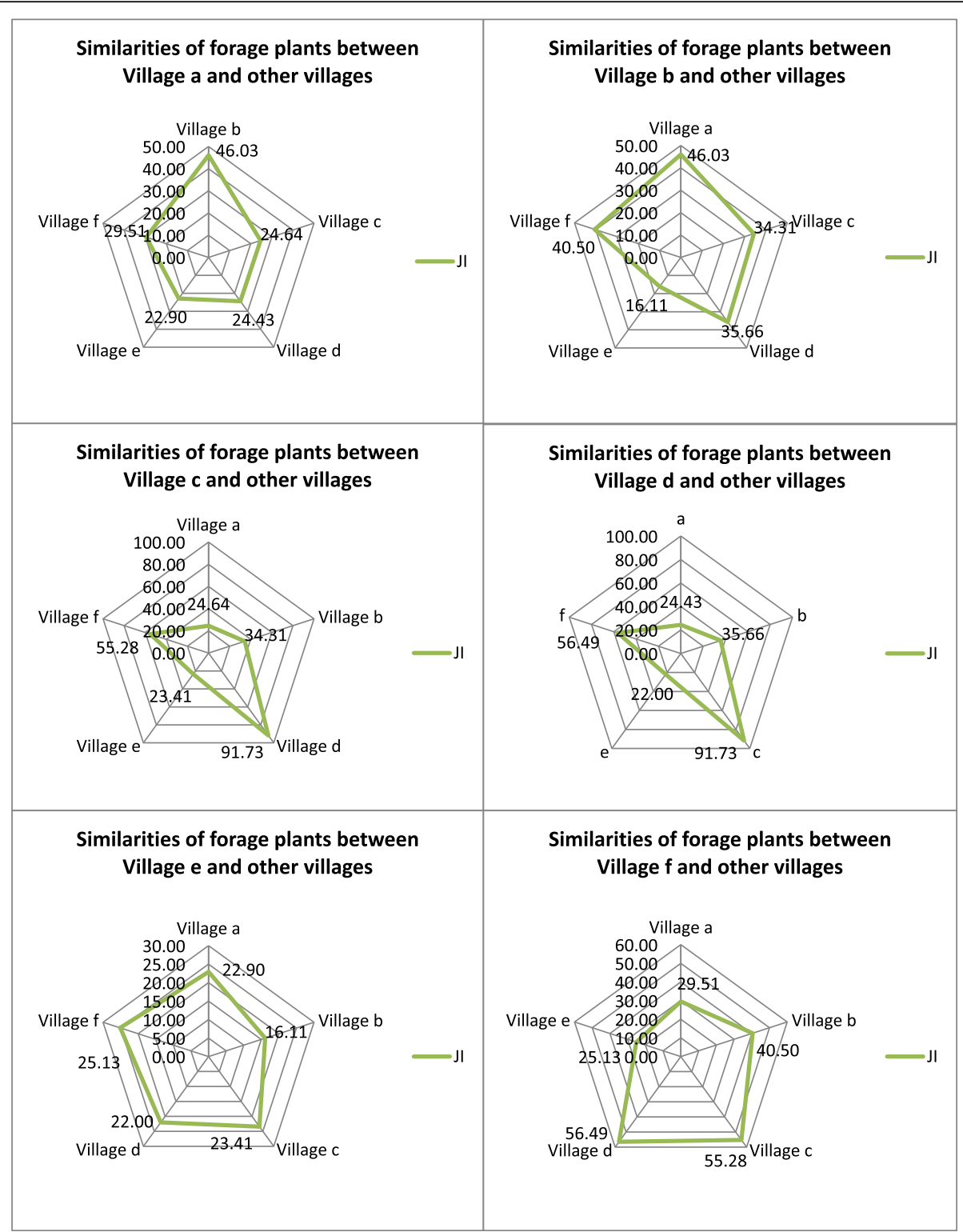

Fig. 3 Similarities in traditional forage plants between six villages

Effects of gender, age, education level, and occupation on traditional forage plant knowledge

The distribution of traditional forage plant knowledge among local populations varies with regard to gender, age, education level, and occupation. As shown in Tables 5 and 6 , knowledge level is significantly correlated with gender $(P<0.05)$, age $(P<0.05)$, education level $(P<$ $0.05)$, and occupation $(P<0.05)$. Among 315 people interviewed in this survey, there were 165 males and 150 females, accounting for $52 \%$ and $48 \%$ of the total, respectively. Males have more knowledge of traditional forage plants than do females $(P=0.005$, pairwise comparison). The mean number of traditional forage plant species reported increased by age group: 15.196 for the respondents under 19 years old, 42.472 for respondents 20-39 years old, 49.826 for the respondents $40-59$ years old, and 51.352 for the respondents over 60 years old. The respondents over 40 years old know the most about traditional forage plant species, but there is no significant difference between 40-59 years old respondents and respondents over 60 years old regarding the mean number of plant species reported $(P=0.660$, pairwise comparison). The respondents between 20 and 39 years old know less, and respondents under 19 years old know the least. From the perspective of education level, the uneducated population (illiterate) knows the most about traditional forage plant species (mean number 52.440) and have the most abundant knowledge $(P$ value $\leq 0.05$, 


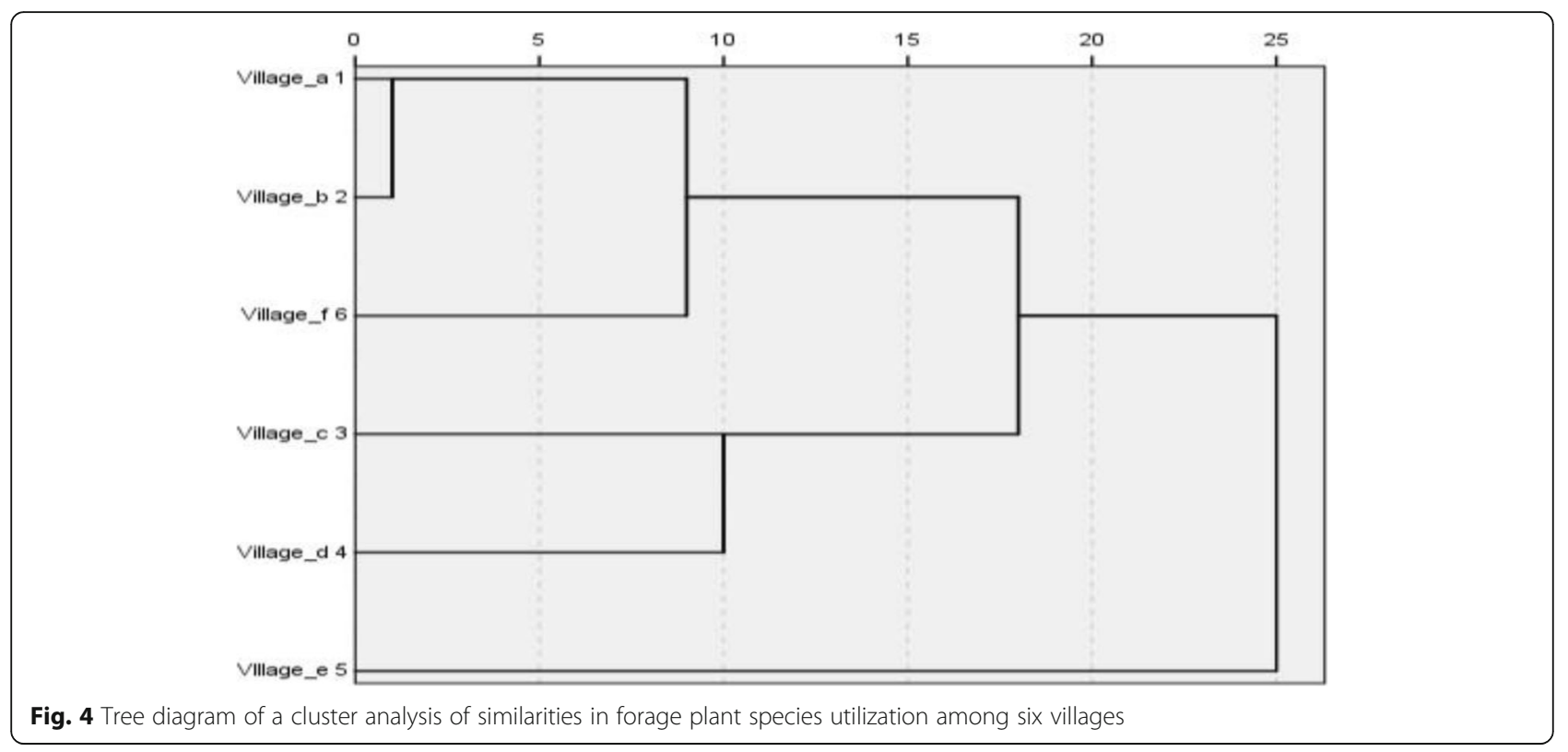

pairwise comparison of this population to people with an education level of primary school, middle school, high school, technical secondary school, or above) in terms of the number of forage plant species reported. From the perspective of occupation, migrant workers (mean 37.488) have relatively less knowledge of traditional forage plants, while students (mean 17.417) have the least knowledge $(P$ value $<0.001$, pairwise comparison of students to people of other occupations). In summary, males over the age of 40 and with a low education level have more understanding and a rich knowledge of traditional forage plants, while students and those under 19 years old have less knowledge regarding traditional forage plants and livestock feeding.

\section{Discussion}

Several years after relocation, new natural and human environments that differ from the place of emigration can gradually change the utilization behavior and the knowledge of ecological migrants towards plant resources [24, 35]. The diversity of traditional forage plant resources and related knowledge retained by ecological immigrants may significantly change after migration. Even though ecological migrants still apply traditional knowledge in their livelihood after migration, knowledge inheritance becomes a concern. The diversity of traditional forage plant resources used by immigrants refers to the types, quantity, and parts of traditional forage plants that can be used as forage $[2,9,36]$, which depends, to a certain extent, on the distribution of forage plant resources around the area they live. The related knowledge includes the knowledge about forage forms of, seasonal use of, suitable livestock for traditional forage plants, and classification of pasture habitats [1, 5, 8, 37-40], which is affected by certain cultural factors, such as livestock rearing and breeding habits, as well as traditional techniques. Previous studies have concluded that the factors affecting traditional plant resources and associated knowledge include the natural environment (ecosystem [41]), traditional culture [42], customs and habits [43], the theoretical system of traditional food and medicine [10,44], traditional production and lifestyle $[45,46]$, and degree of modernization [47, 48]. The present study found that the diversity of traditional forage plants shows both differences and similarities between immigration and emigration locations and that the degree of preservation is closely related to the ecological environment, traditional culture, customs and habits, production and lifestyle [49-51].

Forage plant resources in Ningxia have a diverse distribution due to Ningxia's complex geomorphological structure: the Liupan Mountain water conservation forest in the south, Loess Plateau and Jianshan Basin in the midsouth area, alluvial plain of the Yellow River in the midnorth, and semiarid desertified area partially covering central Ningxia. Diverse ecological environments have resulted in diverse vegetation types and plant species in Ningxia. Throughout history, Ningxia has been a mixed agricultural-pastoral area. Immigration villages a, b, c, and $\mathrm{d}$ in Hongsibu District of Ningxia are in a desert steppe desertification control area in central Ningxia. Therefore, the forage plants used by the villagers have psammophyte, xerophyte, and halophyte characteristics. Village $f$ is located near the emigration area in a loess hilly region where the vegetation possesses typical xerophyte and halophyte characteristics. Village $\mathrm{e}$ is located near the 
Table 5 Knowledge of forage plants based on the characteristics of respondents from five villages

\begin{tabular}{|c|c|c|c|c|}
\hline Characters & Total number of respondents & Average kinds of the forage plants mentioned & $R F C=\frac{F C_{S}}{N}$ & $p$ value \\
\hline Gender & & & 4844.385, df = 1 & 0.000 \\
\hline Male & 165 & 45.153 & & \\
\hline Female & 150 & 37.612 & & \\
\hline Age range & & & $10789.559, \mathrm{df}=3$ & 0.000 \\
\hline$\leq 19$ & 28 & 15.196 & & \\
\hline $20-39$ & 85 & 42.472 & & \\
\hline $40-59$ & 127 & 49.826 & & \\
\hline$\geq 60$ & 75 & 51.352 & & \\
\hline Education Level & & & $1758.473, d f=3$ & 0.002 \\
\hline Illiteracy & 128 & 52.440 & & \\
\hline Primary & 119 & 39.951 & & \\
\hline Middle & 49 & 42.831 & & \\
\hline Higher & 19 & 22.529 & & \\
\hline Occupation & & & $984.535, \mathrm{df}=6$ & 0.013 \\
\hline Village heads & 12 & 64.750 & & \\
\hline Farmers & 244 & 43.630 & & \\
\hline businessmen & 5 & 52.600 & & \\
\hline Migrant workers & 19 & 37.488 & & \\
\hline Village doctors & 5 & 45.800 & & \\
\hline Students & 23 & 17.417 & & \\
\hline Other else & 7 & 47.571 & & \\
\hline
\end{tabular}

emigration area in a forest where the vegetation possesses typical damp (shady) and semidamp (semishady) mountain plant characteristics. In this study, the immigrants in four villages all reported more than 100 forage plant types, but the vegetation types vary among villages. The villagers in Villages $\mathrm{c}, \mathrm{d}$, and $\mathrm{f}$ are good at raising sheep; hence, they listed a variety of psammophytic, xerophytic, and halophytic plants and knew many forage plants for sheep in semiarid areas. Moreover, the plants reported by Villages $c$ and $d$ are highly similar but very different from those reported by Village $f$, indicating a homologous relationship between immigrants from Villages $\mathrm{c}$ and $\mathrm{d}$. The villagers in Village e are good at raising cattle, and their "Jingyuan Yellow Cattle" is a well-known local geographical indication product. The local forage plant species reported by the villagers are highly diverse, and the forage plant knowledge of the villagers is mostly related to the plants that cattle like to eat. This can be attributed to the unique forest landscape and plant resources in the area as well as to traditional cattle-raising experience and knowledge. In conclusion, the diversity of traditional forage plants reported is related to the regional distribution of plant resources. However, we also found a concerning problem. Villages $\mathrm{a}$ and $\mathrm{b}$, who migrated from a forest area and loess hilly area to a semiarid desertified area, experienced prominent changes in the natural environment and forage plant resources. The villagers in the two villages that immigrated more than $200 \mathrm{~km}$ reported a significantly smaller number of forage plants. The long-distance migration of immigrants results in a lack of availability of the biological resources similar to those they had access to in their home area, resulting in changes in the land and biological resource types they have long relied on [52]. For immigrants in environments that lack the biological resources similar to those in their home area, they no longer mention and use these resources; therefore, the specific knowledge associated with these resources will gradually be forgotten $[53,54]$.

To investigate forage knowledge, the villagers were asked to fully describe the plant parts (e.g., leaves, stems, roots, fruits, or inflorescence) that can be used for feeding livestock [55] and palatability (e.g., amount, juicy texture, sweet taste, bitter taste) [2]. The villagers provided information regarding the different adaptive strategies of feeding cattle and sheep in spring, summer, autumn, and winter [4]. In addition, the details regarding how to use the plants as forage were explained. Two forms, including green tender forage and green hay, were described for further classification of forage plants [56], which is of great value to manual management of 
Table 6 Pairwise comparisons of the number of traditional forage plant species reported

\begin{tabular}{|c|c|c|c|}
\hline Characters & Mean difference & S.t.d. error & $p$ value \\
\hline \multicolumn{4}{|l|}{ Gender (i-j) } \\
\hline "Male"-"Female" & 7.541 & 2.647 & 0.005 \\
\hline \multicolumn{4}{|l|}{ Age $(i-j)$} \\
\hline$" \geq 60 "-" \leq 19 "$ & 36.156 & 4.580 & 0.000 \\
\hline " $\geq 60 "-" 20-39 "$ & 8.880 & 3.559 & 0.014 \\
\hline$" \geq 60 "-" 40-59 "$ & 1.526 & 3.463 & 0.660 \\
\hline \multicolumn{4}{|l|}{ Education level (i-j) } \\
\hline "Illiteracy"-"Primary" & 12.490 & 3.032 & 0.000 \\
\hline "Illiteracy"-"Middle" & 9.609 & 3.673 & 0.010 \\
\hline "Illiteracy"-"Higher" & 29.911 & 4.981 & 0.000 \\
\hline \multicolumn{4}{|l|}{ Occupation (i-j) } \\
\hline "Students"-"Village heads" & -47.333 & 6.829 & 0.000 \\
\hline "Students"--Farmers" & -26.213 & 4.413 & 0.000 \\
\hline "Students"-"businessmen" & -35.183 & 9.369 & 0.000 \\
\hline "Students"-"Migrant workers" & -20.071 & 6.256 & 0.002 \\
\hline "Students"-"Village doctors" & -28.383 & 9.369 & 0.003 \\
\hline "Students"-"Other else" & -30.155 & 8.223 & 0.000 \\
\hline
\end{tabular}

forage resources [57]. Particularly, the important effects of some forage plants were summarized [58], which is very valuable. In the interviews, it was learned that some plants can increase fat, act as aphrodisiacs, promote lactation, and reduce "internal heat." Plants that can significantly increase fat in livestock include Krascheninnikovia ceratoides, Melilotus albus, M. ruthenica, Agriophyllum squarrosum, Persicaria vivipara, Artemisia gmelinii, Artemisia desertorum, and Ajania achilleoides. Plants with aphrodisiac and lactation-promoting effects include the following. The juicy branches of Artemisia frigida after the leaves are withered in winter and spring have fattening, fat-retaining, aphrodisiac, and lactationpromoting effects. Lespedeza davurica, Lespedeza potaninii, and Glycyrrhiza uralensis plants can be made into hay at their flowering stage, which serves as good forage for lambing ewes and weak livestock to increase fat and lactation in winter and spring. The medicinal plants with internal heat-reducing and body-strengthening effects are Rheum tanguticum, Rheum palmatum, and Rumex crispus. In summer, these three plants can be boiled in water to produce liquid medicine that can reduce internal heat and relieve summer heat in cattle and sheep. Corispermum patelliform plants can be made into highly nutritional hay to feed lambs and sick sheep in winter. The knowledge above was summarized and inherited from long-term production practice and is a precious treasure that deserves to be recorded and studied.

Some villagers reported that the meat from sheep often fed with Allium mongolicum and Allium polyrhizum tastes better and is free of a mutton smell. Some villagers mentioned that cattle like Convolvulus arvensis plants best. As the local folk adage says "Bitter Vine, donkeys don't eat, horses don't watch, old cattle come to pull as long noodles, and goats and sheep love to eat"; donkeys and horses do not like "Bitter Vine" (local name for $C$. arvensis), but cattle like it as much as the local people like long noodles (a kind of pasta that the local people generally like to eat). Several other plants can be used as forage for cattle and sheep only at the early growing period and become toxic and inedible after fruiting, including Lappula myosotis, Lappula squarrosa subsp. Heteracantha, and Xanthium strumarium subsp. sibiricum. The green tender plants of these three species are good forage for cattle and sheep but become harmful when the seeds mature because the bristles on the edges of the seeds easily stick to the body. In addition, sheep occasionally eats the flowers of $A$. mongolicus, as mentioned earlier. However, A. mongolicus is an important sand-fixing and ancient plant species in semiarid desertified land and a nationally key protected plant. Therefore, villagers should protect this plant from damage during sheep grazing. The knowledge above is of great significance in the development and production of animal husbandry.

Immigrants can make full use of forage plant resources to develop animal husbandry [59], which provides a reliable livelihood for local villagers [60-62]. Cattle and sheep are the main local livestock, playing an important role in the local diet, festivals, weddings, and other customs $[59,62,63]$. Beef and mutton are the main meat sources in the study area and are used to make local delicacies such as boiled lamb, steamed lamb, stewed snacks, stewed beef and lamb soup, soup bowl, stir-fried stewed meat, and sauced beef [59]. Beef and lamb dishes, "Sangza" (deep-fried twisted noodles), "Youxiang" (cake of flour with salt, fried in sesame oil), and stewed noodle soup are necessities at New Year holidays and other festivals [43]. For local marriage customs including the marriage proposal, tea ceremony, flower-adorning ceremony (i.e., place a beautiful flower in the hair of the woman to confirm the engagement), wedding ceremony, and bride's first home visit after the wedding, sheep are the major gift because they symbolize the success and happiness of the marriage, as said in the local proverb "sheep, wheat, and oil are sent to the bride's home as gifts between the engagement and wedding." For each ceremony, the gifts include a sheep, 100 jin of rice ("jin" is a weight unit commonly used in China, and one jin is equal to $0.5 \mathrm{~kg}$ ), sugar, tea leaves, large steamed buns, each weighing 0.5 jin, and "Youxiang," etc. [64]. As seen with these customs, raising and breeding cattle and sheep, as well as possessing related forage plant knowledge, are critical in local life. Because of their traditional 
customs associated with cattle and sheep, the livelihood of ecological immigrants still depends on raising and breeding cattle and sheep even after relocation [65]. Therefore, protecting the traditional culture of immigrants and preserving traditional knowledge associated with biodiversity synergistically promote each other.

Human factors are key for the inheritance of traditional forage plant knowledge. The age, gender, education level, and occupation of ecological immigrants are factors commonly considered in research [66]. In this study, the number of traditional forage plant species reported by respondents was positively correlated with age. Older people could enumerate more forage plant species than could young people, suggesting that elders play a key role in preserving traditional forage plant knowledge and that young people have less understanding of their ancestral homeland after ecological migration. This indicates that there is a disruption risk regarding traditional knowledge inheritance. In the history of local animal husbandry, males have played a more important role and have more knowledge of traditional feeding plants than do females. However, with the livelihood transformation from farming and livestock to non-farming jobs, increasingly more males are pursuing non-farming jobs, and tradition knowledge is gradually being lost. In addition, the number of traditional forage plant species reported by respondents was negatively correlated with education level. The uneducated population (illiterate) had the most abundant knowledge of traditional forage plants, which decreased in the respondents with a higher education level. A possible reason is that uneducated immigrants, who cannot read or write, inherit the traditional knowledge of forage plants through listening and memorizing, while educated immigrants choose non-farming jobs in cities and no longer rely on the traditional farming industry like older generations. This causes a disruption in retaining and passing traditional forage plant knowledge. With regards to occupation, village cadres, knowledgeable masters of local livestock farming, businessmen buying and selling cattle and sheep, and local grassroots doctors have the most knowledge of forage plants and provide key information for the investigation of traditional forage plants. These people play important roles in the protection and inheritance of traditional forage plant knowledge, and they should be supported and helped jointly by the local government, science and technology sectors, forestry and grassland sectors, and industrial associations through incentive measures.

\section{Conclusions}

This study investigated traditional forage plant species and associated traditional knowledge learned and mastered by the villagers who moved to four ecological immigrant villages and those who still live in two villages near the emigration area in the Hongsibu District of Ningxia. Detailed information regarding 224 traditional forage plants were provided, including local names, plant parts commonly used as forage, forage form, and livestock for which the plant is suitable to feed. The value and utilization knowledge of local important forage plants were analyzed. In addition, the differences in the types and number of traditional forage plant species and related knowledge reported during the survey were compared among the ecological immigrants from different areas. Moreover, the effects of gender, age, education level, and occupation on traditional forage plant knowledge were analyzed. We concluded that forage plant resources in the ecological immigration area surveyed is highly diverse and abundant, and forage plant species vary among the forest areas, loess hilly areas, and semiarid desertified areas. Due to different biodiversity backgrounds and living environments, there are differences in the diversity of traditional forage plants and related knowledge retained by ecological immigrants from different emigration areas. Immigrants who move only a short distance well retain the diversity of traditional forage plant sources and related knowledge. In contrast, immigrants who migrate a long distance experience remarkable changes in natural resources and thus have less knowledge regarding the traditional forage plants at the new location. The knowledge of forage plants is being forgotten and abandoned by the younger generation. The changes above may be attributed to multiple factors including changes in the distribution of forage plant resources, livestock farming traditions, and customs. Males, elders, people with less education, and key figures in the villages know the most about traditional forage plants. We suggest that targeted measures should be taken to record and protect forage plant resources and knowledge retained and passed on by ecological immigrants, promoting regional biodiversity preservation and sustainable development.

\footnotetext{
Acknowledgments

We are grateful for the generous support from the people's governments of Hongsibu District, Haiyuan County, and Jingyuan County and all townships where the investigated villages are located, as well as village cadres and villagers of all villages surveyed in this study. We thank Weichun Qin from the Luoshan Administration Bureau of Ningxia and Keku Dong from the Ningxia Liupan Mountain Administration Bureau for providing valuable local information and documents. We thank Wenzhao Tian, Ling Yang, and Yue Wang (Ecology majors at the College of Life and Environmental Sciences, Minzu University of China), Na Li and Xinpeng Zou (Biology majors), Jie Pan (Biotechnology major), and Xuechun Yang (Pharmaceutical Engineering major) for their help with the household survey. We thank Mr. Kechang Li, the former head of the Ningxia Grassland Workstation, Prof. Sheng Qiang from Nanjing Agricultural University, and Dr. Bo Liu from Minzu University of China for their help in plant identification. We also thank the Springer Nature for assistance with English translation and editing services.
} 


\section{Ethics approval and consent of participants}

Informed consent was verbally obtained from all participants prior to the study, which is in accordance with the ethical guidelines adopted by the International Society of Ethnobiology (2008).

\section{Authors' contributions}

DX conceived and designed the study. YM conducted the field research and data analysis and drafted the manuscript in Ningxia. QZ conducted plant identification and verification. YM, DM, and QW performed the statistical analysis. DX, BL, QW, and JF carefully revised the manuscript. All authors have read and approved the final draft.

\section{Funding}

This study was funded by the Project for Developing Top-tier University and Top-tier Disciplines of Minzu University of China (No. YLDX01013), the Project for Developing Ecology as a Top-tier Discipline of Minzu University of China (No. Yldxxk201819), the Independent Scientific Research Project of Doctoral Students of Minzu University of China (project No. 181060), and the Special Project of "Biodiversity Protection" jointly supported by the Ministry of Ecology and Environment and the Ministry of Finance of China(No. 2019HJ2096001006).

\section{Availability of data and materials}

The analyzed data are incorporated in the research article.

\section{Consent for publication}

Not applicable.

\section{Competing interests}

The authors declare they have no competing interests.

\section{Author details}

'College of Life and Environmental Sciences, Minzu University of China, Beijing 100081, People's Republic of China. ${ }^{2}$ Ningxia Forestry Research Institute, Yinchuan 750021, Ningxia, People's Republic of China. ${ }^{3}$ College of Mathematics and Information Science, North Minzu University, Yinchuan 750021, Ningxia, People's Republic of China. ${ }^{4}$ College of Resources and Environment, Ningxia University, Yinchuan 750021, Ningxia, People's Republic of China.

Received: 1 September 2019 Accepted: 15 October 2019

Published online: 16 December 2019

\section{References}

1. Berkes F, Colding J, Folke C. Rediscovery of traditional ecological knowledge as adaptive management. Ecol Appl. 2000;10(5):1251-62 https://doi.org/10. 1890/1051-0761(2000)010[1251:ROTEKA]2.0.CO:2

2. Harun N, Chaudhry AS, Shaheen S, Ullah K, Khan F. Ethnobotanical studies of fodder grass resources for ruminant animals, based on the traditional knowledge of indigenous communities in Central Punjab Pakistan. J Ethnobiol Ethnomed. 2017;13:56 https://doi.org/10.1186/s13002-017-0184-5.

3. Kakinuma K, Takatsuki S. Applying local knowledge to rangeland management in northern Mongolia: do "narrow plants" reflect the carrying capacity of the land? Pastoralism Res Policy Pract. 2012;2(1):23 https://doi. org/10.1186/2041-7136-2-23.

4. Naah JBSN. Investigating criteria for valuation of forage resources by local agro-pastoralists in West Africa: using quantitative ethnoecological approach. J Ethnobiol Ethnomed. 2018;14:62 https://doi.org/10.1186/s13002018-0261-4

5. Volpato G, Nardo AD. The role of Nucularia perrinii Batt. (Chenopodiaceae) in the camel-based Sahrawi social-ecological system. J Ethnobiol Ethnomed. 2017;13:12 https://doi.org/10.1186/s13002-017-0141-3.

6. Freier KP, Bruggemann R, Scheffran J, et al. Assessing the predictability of future livelihood strategies of pastoralists in semi-arid Morocco under climate change. Technol Forecast Soc Change. 2012;79(2):371-82 https:// doi.org/10.1016/j.techfore.2011.07.003.

7. Geng Y, Hu G, Ranjitkar S, et al. Prioritizing fodder species based on traditional knowledge: a case study of mithun (Bos frontalis) in Dulongjiang area, Yunnan Province, Southwest China. J Ethnobiol Ethnomed. 2017:13:24 https://doi.org/10.1186/s13002-017-0153-z.
8. Nunes AT, Lucena RFP, Santos MVF, et al. Local knowledge about fodder plants in the semi-arid region of Northeastern Brazil. J Ethnobiol Ethnomed. 2015;11:12 https://doi.org/10.1186/1746-4269-11-12.

9. Badshah L, Hussain F. Farmers preferences and use of local fodder flora in Tank district, Pakistan. Afr J Biotechnol. 2011;10(32):6062-71.

10. Vogl CR, Vogl-Lukasser B, Walkenhorst M. Local knowledge held by farmers in Eastern Tyrol (Austria) about the use of plants to maintain and improve animal health and welfare. J Ethnobiol Ethnomed. 2016;12:40 https://doi. org/10.1186/s13002-016-0104-0.

11. Xue DY. The latest development for implementation of the Convention on Biological Diversity. Biodiversity Sci. 2017;25(11):1145-6 https://doi.org/10. 17520/biods.2017310,

12. Xue DY. International process for the protection of traditional knowledge under the Convention on Biological Diversity. Guizhou Soc Sci. 2014;292(4): 138-43 https://doi.org/10.13713/j.cnki.cssci.2014.04.023.

13. Khasbagan PSJ. Ethnobotany of forage plants:a case study in Arhorchin Banner of Inner Mongolia. Acta Prataculturae Sin. 2000;9(3):74-81.

14. Tan Y. Resettlement and climate impact: addressing migration intention of resettled people in west China. Aust Geogr. 2017;48(1):97-119 https://doi. org/10.1080/00049182.2016.1266593.

15. Du FC. A review on the ecological resettlement research in Western academic society. Ethno Stud. 2014;2:109-20.

16. Jia YF. Review of benefit evaluation research on ecological migration in China. Resour Sci. 2016;38(8):1550-60 https://doi.org/10.18402/resci.2016.08. 13.

17. Heurtebise JY. Sustainability and ecological civilization in the age of Anthropocene: an epistemological analysis of the psychosocial and "culturalist" interpretations of global environmental risks. Sustainability. 2017; 9(8):1331 https://doi.org/10.3390/su9081331.

18. Liu Y, Guo Y, Zhou Y. Poverty alleviation in rural China: policy changes, future challenges and policy implications. China Agr Econ Rev. 2018;10(2): 241-59 https://doi.org/10.1108/caer-10-2017-0192.

19. Wang Z, Song K, Hu L. China's largest scale ecological migration in the Three-River Headwater Region. Ambio. 2010;39(5):443-6 https://doi.org/10. 1007/s13280-010-0054-z.

20. Huang J, Wang R, Huang J, Wang F, Zhang H. Analysis of the pattern and meaning of ecological immigration in Tarim river middle and lower reaches. Environ Prot Xinjiang. 2004;26(1):71-4.

21. Zhang L, Wang X, Hou Y, Li C. Synergies between biodiversity conservation and poverty reduction in China. Biodivers Sci. 2015;23(2):271-7 https://doi. org/10.17520/biods.2014241.

22. Ma Y, Yang JB, Wen Q, Xue DY. A study on the changes of traditional knowledge related to biodiversity of ecological immigrants—based on the investigation of immigrant villages in Hongsibu district of Ningxia. Ningxia Soc Sci. 2019:213(1):146-51.

23. Ma Y, Yang JB, Liu HO, Wen Q, Xue DY. Impact of ecological migration on biodiversity-related traditional knowledge. J Minzu U China. 2018;27(4):33-40.

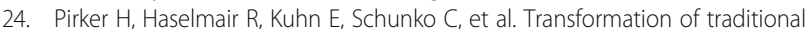
knowledge of medicinal plants: the case of Tyroleans (Austria) who migrated to Australia, Brazil and Peru. J Ethnobiol Ethnomed. 2012;8:44 https://doi.org/10.1186/1746-4269-8-44.

25. Fontefrancesco M, Barstow C, Grazioli F, et al. Keeping or changing? Two different cultural adaptation strategies in the domestic use of home country food plant and herbal ingredients among Albanian and Moroccan migrants in Northwestern Italy. J Ethnobiol Ethnomed. 2019;15:11 https://doi.org/10. 1186/s13002-019-0290-7.

26. Xue X, Wang $T$, Wu W, Sun Q, Zhao C. The desertification development and its causes of agro-pastoral mixed regions in North China. J Desert Res. 2005;25(3):320-8 https://doi.org/10.3321/j.issn: 1000-694X.2005.03.004

27. Li Y, Xu F, Li X. Study on sustainable development of ecological immigrants in Ningxia. Ningxia Soc Sci. 2012;1:29-35.

28. He JW, Zhang RF, Lei QY, et al. Diversity, knowledge, and valuation of plants used as fermentation starters for traditional glutinous rice wine by Dong communities in Southeast Guizhou, China. J Ethnobiol Ethnomed. 2019;15: 20 https://doi.org/10.1186/s13002-019-0299-y.

29. Nunes AT, Cabral DLV, Elba LCA, et al. Plants used to feed ruminants in semi-arid Brazil: a study of nutritional composition guided by local ecological knowledge. J Arid Environ. 2016;135:96-103 https://doi.org/10. 1016/j.jaridenv.2016.08.015

30. Vitalini S, Iriti M, Puricelli C, Ciuchi D, Segale A, Fico G. Traditional knowledge on medicinal and food plants used in Val San Giacomo 
(Sondrio, Italy) —an alpine ethnobotanical study. J Ethnopharmacol. 2013; 145(2):517-29 https://doi.org/10.1016/j.jep.2012.11.024.

31. Bullitta S, Re GA, Manunta MDL, et al. Traditional knowledge about plant, animal, and mineral-based remedies to treat cattle, pigs, horses, and other domestic animals in the Mediterranean island of Sardinia. J Ethnobiol Ethnomed. 2018;14:50 https://doi.org/10.1186/s13002-018-0250-7.

32. Bahru T, Asfaw Z, Demissew S. Ethnobotanical study of forage/fodder plant species in and around the semi-arid Awash National Park, Ethiopia. J Forest Res. 2014;25:445-54 https://doi.org/10.1007/s11676-014-0474-X.

33. Yaseen $\mathrm{G}$, Ahmad M, Sultana $\mathrm{S}$, et al. Ethnobotany of medicinal plants in the Thar desert (Sindh) of Pakistan. J Ethnopharmacol. 2015;163:43-59 https://doi.org/10.1016/j.jep.2014.12.053.

34. Sivasankari B, Anandharaj M, Gunasekaran P. An ethnobotanical study of indigenous knowledge on medicinal plants used by the village peoples of Thoppampatti, Dindigul district, Tamilnadu, India. J Ethnopharmacol. 2014; 153(2):408-23 https://doi.org/10.1016/j.jep.2014.02.040.

35. Waldstein A. Diaspora and health? Traditional medicine and culture in a Mexican migrant community. Int Migr. 2008;46(5):95-117 https://doi.org/10. 1111/j.1468-2435.2008.00490.x.

36. Morgan WTW. Ethnobotany of the Turkana: use of plants by a Pastoral people and their livestock in Kenya. Econ Bot. 1981;35(1):96-130 https://doi. org/10.1007/bf02859220.

37. Sansoucy R. Livestock: a driving force for food security and sustainable development. World Anim Rev. 1995;84(85):5-17 https://doi.org/10.1016/j. tetlet.2009.11.059.

38. Abioye A, Zaid Y, Egberongbe H. Documenting and disseminating agricultural indigenous knowledge for sustainable food security: the efforts of agricultural research libraries in Nigeria. Libri. 2014;64(1):75-84 https://doi. org/10.1515/libri-2014-0007.

39. Nogoonmuqir T, Soyolt K. Investigation and analysis of wild forage plant resource in nomadic grazing system of Arhorqin pastures-a case study of Hundleng summer base. Chin Wild Plant Resour. 2017;36(2):66-84.

40. Molnár Z. Classification of pasture habitats by Hungarian herders in a steppe landscape (Hungary). J Ethnobiol Ethnomed. 2012;8:28 https://doi. org/10.1186/1746-4269-8-28.

41. Athayde S, Silvalugo J. Adaptive strategies to displacement and environmental change among the Kaiabi indigenous people of the Brazilian Amazon. Soc Nat Resour. 2018;Online(2):1-17 https://doi.org/10.1080/ 08941920.2018.1426801.

42. Gadgil M, Berkes F, Folke C. Indigenous knowledge for biodiversity conservation. Ambio. 1993;22(2-3):151-6 https://doi.org/10.1111/issj.12038.

43. Gao HY. A study of the folk culture of the spring festival in Xihaigu of Ningxia. Modern Wom: Theor Front. 2013;1:114-7.

44. Depo $\mathrm{Y}$, Junfeng J, Yongping $\mathrm{H}$, et al. Theoretical system of ecocare and its applications in health care and product development of traditional Chinese medicine. World Sci Tech. 2010;12(2):155-9 https://doi.org/10.1016/S18763553(11)60013-6.

45. Gómez-Baggethun E, Mingorría S, Reyes-García V, Calvet-Mir L, Montes C. Traditional ecological knowledge trends in the transition to a market economy: empirical study in the Doñana natural areas. Conserv Biol. 2010; 24(3):721-9 https://doi.org/10.1111/j.1523-1739.2009.01401.X.

46. Chepape RM, Mbatha KR, Luseba D. Local use and knowledge validation of fodder trees and shrubs browsed by livestock in Bushbuckridge area, South Africa. Ghanaian Popul. 2014;77:20-47.

47. Thomas E. The impact of traditional lifestyle, provenance and contact history on plant use knowledge and management: a cross-cultural comparison of two small-scale societies from the Bolivian Amazon. Hum Ecol. 2012;40(3):355-68 https://doi.org/10.1007/s10745-012-9488-1.

48. Quinlan MB, Quinlan RJ. Modernization and medicinal plant knowledge in a Caribbean horticultural village. Med Anthropol Quart. 2007;21(2):169-92 https://doi.org/10.1525/MAQ.2007.21.2.169.

49. Bollig M, Schulte A. Environmental change and pastoral perceptions: degradation and indigenous knowledge in two African pastoral communities. Hum Ecol. 1999;27(3):493-514 https://doi.org/10.1023/a: 1018783725398.

50. Linstädter A, Kemmerling B, Baumann G, et al. The importance of being reliable-local ecological knowledge and management of forage plants in a dryland pastoral system (Morocco). J Arid Environ. 2013;95:30-40 https://doi. org/10.1016/j.jaridenv.2013.03.008

51. Naah JBSN, Guuroh RT. Factors influencing local ecological knowledge of forage resources: ethnobotanical evidence from West Africa's savannas. J
Environ Manage. 2017;188:297-307 https://doi.org/10.1016/j.jenvman.2016 11.064 .

52. Medeiros PM, Soldati GT, Alencar NL, et al. The use of medicinal plants by migrant people: adaptation, maintenance, and replacement. Evid-Based Complementray Altern Med. 2012;2012(2):807452 https://doi.org/10.1155/ 2012/807452.

53. Ceuterick M, Vandebroek I, Pieroni A. Resilience of Andean urban ethnobotanies: a comparison of medicinal plant use among Bolivian and Peruvian migrants in the United Kingdom and in their countries of origin. J Ethnopharmacol. 2011;136(1):27-54 https://doi.org/10.1016/j.jep.2011.03.038.

54. Volpato G, Godínez D, Beyra A, et al. Uses of medicinal plants by Haitian immigrants and their descendants in the Province of Camagüey. Cuba. J Ethnobiol Ethnomed. 2009;5:1-9 https://doi.org/10.1186/1746-4269-5-16.

55. Ahmad F, Khan M, Ahmad M, et al. Ethnomedicinal uses of grasses in the salt range region of Northern Pakistan. J Med Plants Res. 2010;4(5):362-9 https://doi.org/10.1007/s12272-010-0320-4.

56. Zollitsch W, Kristensen T, Krutzinna C, MacNaeihde F, Younie D. Feeding for health and welfare: the challenge of formulating well-balanced rations in organic livestock production. In: Vaarst M, Roderick S, Lund V, Lockeretz W, editors. Animal Health and Welfare in Organic Agriculture. Wallingford: CABI Publishing; 2004. p. 329-56. https://doi.org/10.1079/9780851996684.0329.

57. Plieninger T, Wilbrand C. Land use, biodiversity conservation, and rural development in the dehesas of Cuatro Lugares, Spain. Agroforest Syst. 2001; 51(1):23-34 https://doi.org/10.1023/a:1006462104555.

58. Pieroni A, Howard P, Volpato G, et al. Natural remedies and nutraceuticals used in ethnoveterinary practices in Inland Southern Italy. Vet Res Commun. 2004;28(1):55 https://doi.org/10.1023/b:verc.0000009535.96676.eb.

59. Ma Z, Hu J, Ma Z. The principal animal husbandry in South Ningxia_-iseussion of cattle and sheep (goat) development. Agric Res Arid Area. 1995;13(3):148-54

60. Yang M, Li T, Mi W, Zhou M, Wang T. Ecological fragile assessment and regional development model in restrict development area of Ningxia. Bull Soil Water Conserv. 2014;34(4):236-42 https://doi.org/10.13961/j.cnki.stbctb. 2014.04.060.

61. Zhang H, Si J, Zhang R. Study on optimization and adjustment of planting structure in Yangsi Irrigation district of Hongsibu, Ningxia. People Yellow River. 2011;33(5):78-9.

62. Xia H. Comparative advantages and countermeasures of constructing Halal beef and mutton industrial base in Ningxia from an international perspective. Econ Forum. 2011;3:105-8.

63. Ding C, Sha B. Hui people's tea drinking customs. China Food. 2008;5:37-9.

64. Liang Z, Qi G, Li X. Isomorphic development: government guidance and peasant participation in the change of Hui villages. J Southwest $U$ Nationalities (Hum Soc Sci Edit). 2014;1:49-53.

65. Yang G, Tan J. A discussion of the relations between the Hui traditional culture and economic development. J Anhui Agri U. 2002;11(5):60-2.

66. Mao S, Shen Y, Deng H, et al. Distribution pattern of traditional ecological knowledge on plant utilization among major minority peoples in Guizhou, China. Int J Sust Dev World. 2018;7644:1-8 https://doi.org/10.1080/ 13504509.2018.1464978.

\section{Publisher's Note}

Springer Nature remains neutral with regard to jurisdictional claims in published maps and institutional affiliations.

Ready to submit your research? Choose BMC and benefit from:

- fast, convenient online submission

- thorough peer review by experienced researchers in your field

- rapid publication on acceptance

- support for research data, including large and complex data types

- gold Open Access which fosters wider collaboration and increased citations

- maximum visibility for your research: over $100 \mathrm{M}$ website views per year

At BMC, research is always in progress.

Learn more biomedcentral.com/submission 